\title{
A UNIFIED THEORY FOR THE ATMOSPHERES OF THE HOT AND VERY HOT JUPITERS: TWO CLASSES OF IRRADIATED ATMOSPHERES
}

\author{
J. J. FORTNEY ${ }^{1,2,3}$ \\ Space Science and Astrobiology Division, Mail Stop 245-3, NASA Ames Research Center, Moffett Field, CA 94035; jfortney@ucolick.org \\ K. LODDERS \\ Planetary Chemistry Laboratory, Department of Earth and Planetary Sciences, Washington University, St. Louis, MO 63130
}

AND

M. S. Marley and R. S. Freedman ${ }^{2}$

Space Science and Astrobiology Division, Mail Stop 245-3, NASA Ames Research Center, Moffett Field, CA 94035

Received 2007 September 4; accepted 2007 December 7

\begin{abstract}
We highlight the importance of gaseous TiO and VO opacity on the highly irradiated close-in giant planets. The dayside atmospheres of these planets naturally fall into two classes that are somewhat analogous to the M- and L-type dwarfs. Those that are warm enough to have appreciable opacity due to TiO and VO gases we term "pM class" planets, and those that are cooler we term "pL class" planets. We calculate model atmospheres for these planets, including pressure-temperature profiles, spectra, and characteristic radiative time constants. We show that $\mathrm{pM}$ class planets have temperature inversions (hot stratospheres), appear "anomalously" bright in the mid-infrared secondary eclipse, and feature molecular bands in emission rather than absorption. From simple physical arguments, we show that they will have large day/night temperature contrasts and negligible phase shifts between orbital phase and thermal emission light curves, because radiative timescales are much shorter than possible dynamical timescales. The $\mathrm{pL}$ class planets absorb incident flux deeper in the atmosphere where atmospheric dynamics will more readily redistribute absorbed energy. This will lead to cooler day sides, warmer night sides, and larger phase shifts in thermal emission light curves. The boundary between these classes ( $\sim 0.04-0.05$ AU from a Sun-like primary for solar composition) is particularly dependent on the incident flux from the parent star, and less so on other factors. We apply these results to several planets and note that the eccentric transiting planets HD $147506 \mathrm{~b}$ and HD 17156b alternate between the classes. Thermal emission in the optical from pM class planets is significant redward of $400 \mathrm{~nm}$, making these planets attractive targets for optical detection. The difference in the observed day/night contrast between $v$ And b (pM class) and HD $189733 \mathrm{~b}$ (pL class) is naturally explained in this scenario.
\end{abstract}

Subject headings: planetary systems — radiative transfer

\section{INTRODUCTION}

The blanket term "hot Jupiter" or even the additional term "very hot Jupiter" belies the diversity of these highly irradiated planets. Each planet likely has its own unique atmosphere, interior structure, and accretion history. The relative amounts of refractory and volatile compounds in a planet will reflect the parent star abundances, nebula temperature, total disk mass, location of the planet's formation within the disk, duration of its formation, and its subsequent migration (if any). This accretion history will give rise to differences in core masses, total heavy elements abundances, and atmospheric abundance ratios. Given this incredible complexity, it is worthwhile to first look for physical processes that may be common to groups of planets.

In addition to a mass and radius, one can further characterize a planet by studying its atmosphere. The visible atmosphere is a window into the composition of a planet and contains clues to its formation history (e.g., Marley et al. 2007). Of premier importance in this class of highly irradiated planets is how stellar insolation affects the atmosphere, as this irradiation directly af-

\footnotetext{
1 Spitzer Fellow.

2 Carl Sagan Center, SETI Institute, 515 North Whisman Road, Mountain View, CA 94043.

3 Department of Astronomy and Astrophysics, UCO/Lick Observatory, University of California, Santa Cruz, CA 95064.
}

fects the atmospheric structure, temperatures, and chemistry, the planet's cooling and contraction history, and even its stability against evaporation.

Since irradiation is perhaps the most important factor in determining the atmospheric properties of these planets, we examine the insolation levels of the 23 known transiting planets. We restrict ourselves to those planets more massive than Saturn, and hence for now exclude treatment of the "hot Neptune" GJ 436b, which is by far the coolest known transiting planet. Figure 1 illustrates the stellar flux incident on the planets as a function of both planet mass (Fig. 1a) and planet surface gravity (Fig. 1b). In these plots diamonds indicate transiting planets and triangles indicate other interesting hot Jupiters for which Spitzer Space Telescope data exist, but which do not transit.

The first known transiting planet, HD 209458b, is seen to be fairly representative of these planets in terms of incident flux. Planets OGLE-TR-56b and OGLE-TR-132b are somewhat separate from the rest of the group because they receive the highest stellar irradiation. Both orbit their parent stars in less than 2 days and are prototypes of what has been called the class of "very hot Jupiters" (Konacki et al. 2003; Bouchy et al. 2004) with orbital periods less than 3 days. However, orbital period is a poor discriminator between "very hot" and merely "hot," as HD 189733b clearly shows. Labeled a very hot Jupiter on its discovery, due to its short 2.2 day period (Bouchy et al. 2005), HD 189733b 

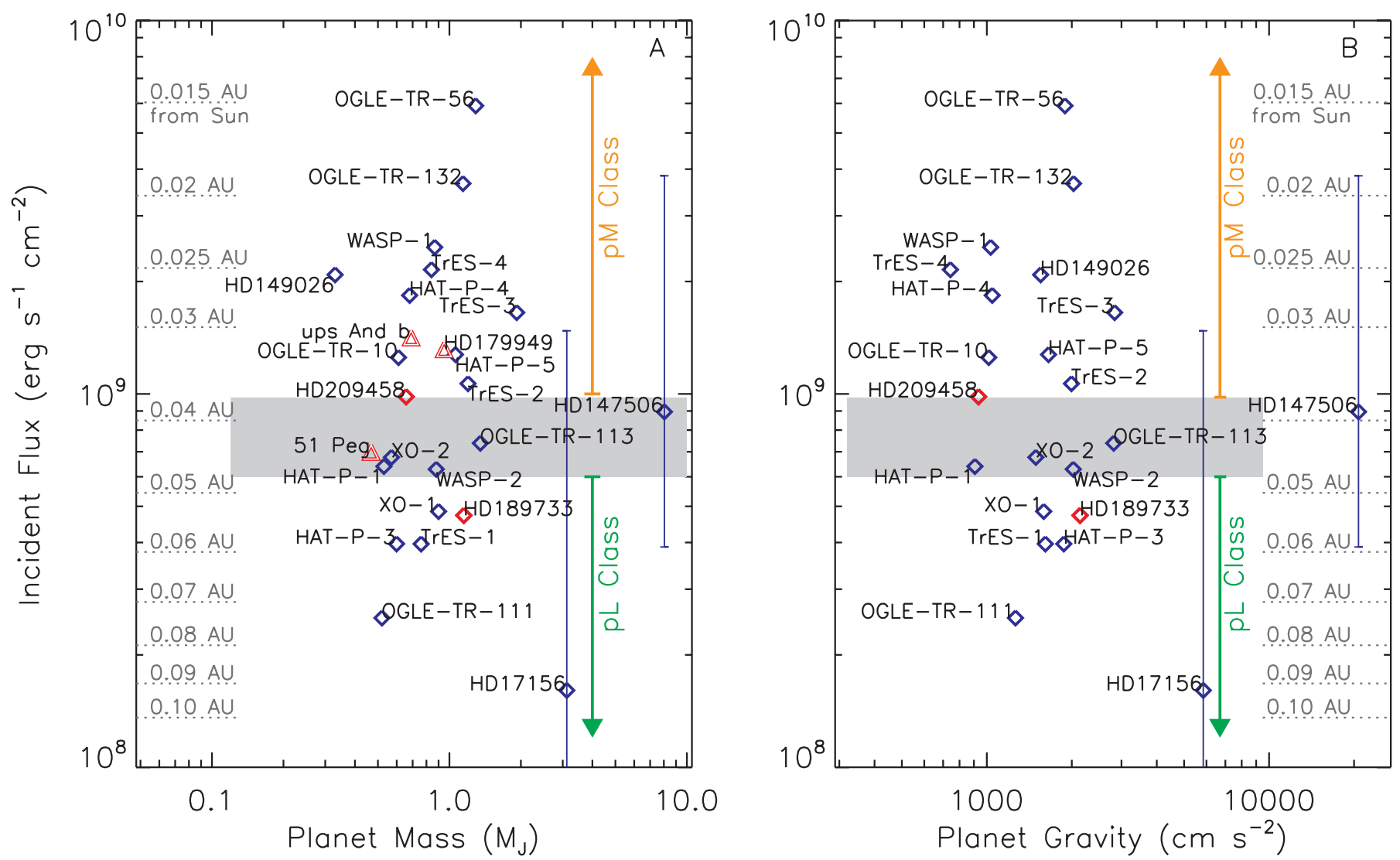

FIG. 1.-Flux incident on a collection of hot Jupiter planets. At left is incident flux as a function of planet mass, and at right as a function of planet surface gravity. In

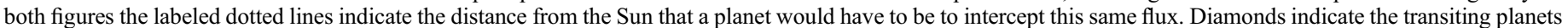

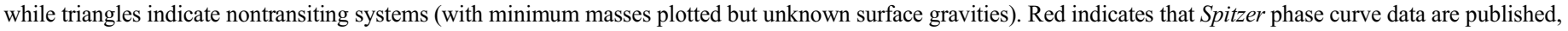

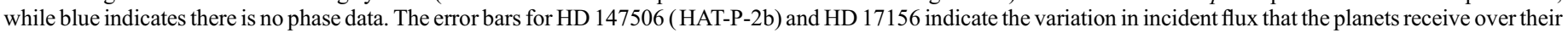

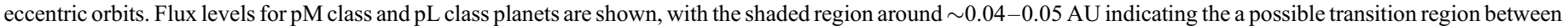
the classes. "Hot Neptune" GJ $436 \mathrm{~b}$ experiences less intense insolation and is off the bottom of this plot at $3.2 \times 10^{7} \mathrm{ergs} \mathrm{s}^{-1} \mathrm{~cm}^{-2}$.

actually receives a comparatively modest amount of irradiation due to its relatively cool parent star. Therefore, perhaps a classification based on incident flux, equilibrium temperature, or other attributes would be more appropriate. In this paper we argue that based on the examination of few physical processes that two classes of hot Jupiter atmospheres emerge with dramatically different spectra and day/night contrasts. Equilibrium chemistry, the depth to which incident flux will penetrate into a planet's atmosphere, and the radiative time constant as a function of pressure and temperature in the atmosphere all naturally define two classes these irradiated planets.

Our work naturally builds on the previous work of Hubeny et al. (2003), who first investigated the effects of $\mathrm{TiO}$ and $\mathrm{VO}$ opacity on close-in giant planet atmospheres as a function of stellar irradiation. These authors computed optical and nearinfrared spectra of models with and without $\mathrm{TiO} / \mathrm{VO}$ opacity. In general, they found that models with $\mathrm{TiO} / \mathrm{VO}$ opacity feature temperature inversions and molecular bands are seen in emission, rather than absorption. Two key questions from the initial Hubeny et al. (2003) investigation were addressed but could not be definitely answered were (1) whether a relatively cold planetary interior would lead to $\mathrm{Ti} / \mathrm{V}$ condensing out deep in the atmosphere regardless of incident flux, thereby removing gaseous $\mathrm{TiO}$ and VO; and (2) if this condensation did not occur, at what irradiation level would $\mathrm{TiO} / \mathrm{VO}$ indeed be lost at the lower atmospheric temperatures found at smaller incident fluxes.

Later Fortney et al. (2006b) investigated model atmospheres of planet $\mathrm{HD} 149026 \mathrm{~b}$, including $\mathrm{TiO} / \mathrm{VO}$ opacity at various metallicities. Particular attention was paid to the temperature of the deep atmosphere pressure-temperature $(P-T)$ profiles (as derived from an evolution model) in relation to the $\mathrm{Ti} / \mathrm{V}$ condensation boundary. Similar to Hubeny et al. (2003), they found a temperature inversion due to absorption by $\mathrm{TiO} / \mathrm{VO}$ and computed near- and mid-infrared spectra that featured emission bands. Using the Spitzer Infrared Array Camera (IRAC) Harrington et al. (2007) observed HD 149026b in secondary eclipse with Spitzer at $8 \mu \mathrm{m}$ and derived a planet-to-star flux ratio consistent with a Fortney et al. (2006b) model with a temperature inversion due to $\mathrm{TiO} / \mathrm{VO}$ opacity. At that point, looking at the work of Fortney et al. (2006b) and especially Hubeny et al. (2003), Harrington et al. (2007) could have postulated that all objects more irradiated than HD 149026b may possess inversions due to $\mathrm{TiO} / \mathrm{VO}$ opacity, but given the single-band detection of HD 149026b, caution was in order. More recently, based on the four-band detection of flux from HD 209458b by Knutson et al. (2008), Burrows et al. (2007b) found that a temperature inversion, potentially due to $\mathrm{TiO} / \mathrm{VO}$ opacity, is necessary to explain this planet's midinfrared photometric data. Based on their new HD 209458b model and the previous modeling investigations, these authors posit that planets warmer than HD $209458 \mathrm{~b}$ may feature inversions, while less irradiated objects such as HD 189733 b do not, and discuss photochemical products and gaseous $\mathrm{TiO} / \mathrm{VO}$ as potential absorbers that may lead to this dichotomy.

We find, as has been previously shown, that those planets that are warmer than required for condensation of titanium ( Ti)- and vanadium (V)-bearing compounds will possess a temperature 
inversion at low pressure due to absorption of incident flux by $\mathrm{TiO}$ and VO, and will appear "anomalously" bright in secondary eclipse at mid-infrared wavelengths. Thermal emission in the optical will be significant (Hubeny et al. 2003; López-Morales $\&$ Seager 2007). Furthermore, here we propose that these planets will have large day/night effective temperature contrasts. We term these very hot Jupiters the pM class, meaning that gaseous $\mathrm{TiO}$ and $\mathrm{VO}$ are the prominent absorbers of optical flux. The predictions of equilibrium chemistry for these atmospheres are similar to dM stars, where absorption by $\mathrm{TiO}, \mathrm{VO}, \mathrm{H}_{2} \mathrm{O}$, and $\mathrm{CO}$ is prominent (Lodders 2002). Planets with temperatures below the condensation curve of $\mathrm{Ti}$ and $\mathrm{V}$ bearing compounds will have a gradually smaller mixing ratio of $\mathrm{TiO}$ and $\mathrm{VO}$, leaving $\mathrm{Na}$ and $\mathrm{K}$ as the major optical opacity sources (Burrows et al. 2000), along with $\mathrm{H}_{2} \mathrm{O}$, and $\mathrm{CO}$. We term these planets the $\mathrm{pL}$ class, similar to the $\mathrm{dL}$ class of ultracool dwarfs. These planets will have relatively smaller secondary eclipse depths in the mid-infrared and significantly smaller day/night effective temperature contrasts. As discussed below, published Spitzer data are consistent with this picture. The boundary between these classes, at irradiation levels (and atmospheric temperatures) where Ti and V may be partially condensed is not yet well defined.

In this paper we begin by discussing the observations to date. We then give an overview of our modeling methods and the predicted chemistry of Ti and V. We calculate $P-T$ profiles and spectra for models planets. For these model atmospheres we then analyze in detail the deposition of incident stellar flux and the emission of thermal flux, and go on to calculate characteristic radiative time constants for these atmospheres. We briefly examine transmission spectra before we apply our models to known highly irradiated giant planets. Before our discussion and conclusions we address issues of planetary classification.

\section{REVIEW OF SPITZER OBSERVATIONS}

\subsection{Secondary Eclipses}

The Spitzer Space Telescope allows astronomers to measure the thermal emission from $\sim 3-30 \mu \mathrm{m}$ from the highly irradiated atmospheres of these extra-solar giant planets (EGPs). This field has progressed quickly from the first observations of secondary eclipses (when the planet passes behind its parent star) by Charbonneau et al. (2005) for TrES-1 and by Deming et al. (2005) for HD 209458b. Once it was clear what Spitzer's capabilities were for these planets, additional observations came quickly. These included secondary eclipses for HD $189733 \mathrm{~b}$ (Deming et al. 2006), HD 149026b (Harrington et al. 2007), GJ 436b (Deming et al. 2007; Demory et al. 2007), and a wealth of new (yet to be published) data for $\sim 10$ other systems. In addition, mid-infrared spectra were obtained for HD $209458 \mathrm{~b}$ (Richardson et al. 2007; Swain et al. 2008) and HD 189733b (Grillmair et al. 2007). In particular, Richardson et al. (2007) claim evidence for emission features in the mid-infrared spectrum. Very recently, Knutson et al. (2008) observed the secondary eclipse of HD 209458b in all four IRAC bands.

One can now begin to examine the secondary eclipse data for trends in planetary brightness temperatures. The brightness temperature, $T_{B}$ is defined as the temperature necessary for a blackbody planet to emit the flux observed in the given observational wavelength or band (Chamberlain \& Hunten 1987). For the published secondary eclipse data as of 2007 June, Harrington et al. (2007) show that the observed dayside brightness temperatures for most transiting planets (TrES-1, HD 189733b, HD 209458b) vary by $\pm 20 \%$ from calculated planetary equilibrium temperatures (when making a uniform assumption for all planets re- garding albedo and flux redistribution). These differences are presumably due to wavelength dependent opacity and varying dynamical redistribution of the absorbed flux. However, HD 149026 b has a $~ 45 \%$ higher brightness temperature than predicted from this relation, meaning that the temperature one measures at $8 \mu \mathrm{m}$ is significantly higher than the planet's equilibrium temperature. This level of infrared emission was predicted by Fortney et al. (2006b) for models of HD 149026b that included a hot stratosphere induced by absorption of stellar flux by $\mathrm{TiO}$ and VO. More recent Knutson et al. (2008) observations of HD 209458b are broadly similar to the one-band detection for HD 149026b. Relatively high brightness temperatures of 1500$1900 \mathrm{~K}$ were observed from 3.6 to $8 \mu \mathrm{m}$, compared to the Harrington et al. (2007) predicted equilibrium temperature of $1330 \mathrm{~K}$. These observations have also been interpreted by Burrows et al. (2007b) as being caused by a temperature inversion (hot stratosphere). As we discuss in $\S 5.1$, HD $209458 \mathrm{~b}$ is in a temperature regime that is difficult to model, where Ti and $\mathrm{V}$ are expected to be partially condensed.

\subsection{Phase Curves}

The observations that best allow us to understand the atmospheric dynamics of these presumably tidally locked planets are those made as a function of orbital phase. Two approaches have been used to measure the thermal emission of these planets away from secondary eclipse. The first is to periodically observe the infrared flux from the combined planet + star system at several times in the planet's orbit. The advantage of periodic short observations is that little telescope time is used. The disadvantage is that since the system is not monitored continuously, systematic uncertainties could be important and may be hard to correct for. There are published data for four planets that have been observed in this way: detections of phase variation for $v$ And $\mathrm{b}$ (Harrington et al. 2006) and HD 179949b (Cowan et al. 2007), and upper limits for HD 209458b, 51 Peg b (Cowan et al. 2007). Alternatively, one could monitor a system continuously over a significant fraction of a planet's orbital period to eliminate any uncertainties induced by having to revisit the target. This method was employed by Knutson et al. (2007) to observe HD 189733b continuously for $33 \mathrm{hr}$, including the transit and secondary eclipse. Large day/night contrasts were found for $\mathrm{pM}$ class planets $v$ And $\mathrm{b}$ (which is consistent with a dark night side) and HD 179949b, whereas pL class planet HD $189733 \mathrm{~b}$ has a day/night $8 \mu \mathrm{m}$ brightness temperature difference of only $\sim 240 \mathrm{~K}$. We return to the Cowan et al. (2007) upper limit for HD 209458b in $\S 8$.

Two additional factors that affect these observations are the brightness of the parent star and the inclination of the planetary orbit. While the brightest planet-hosting stars allow for the largest flux measurements (e.g., $v$ And b), the brightest hot Jupiter systems do not transit. A measurement of the secondary eclipse depth while obtaining a light curve is extremely valuable, because the planet-to-star flux ratio at full planet illumination is thus known, as is the planet's radius from transit observations. The interpretation is then much more straightforward for the transiting systems.

Among the current published data there appear to be two planets that are bright in secondary eclipse, HD 149026b and HD 209458b, compared to other transiting planets, and two planets that appear to have large day/night temperature differences, $v$ And $\mathrm{b}$ and HD $179949 \mathrm{~b}$. We show that these planets, HD 149026b, HD 209458b, $v$ And b, and HD 179949b, are pM class planets, while the less irradiated hot Jupiters (such as HD $189733 \mathrm{~b}$ and TrES-1) are pL class. We now turn to models 
of these classes of planets to examine how and why their atmospheres differ so strikingly.

\section{MODEL ATMOSPHERES}

\subsection{Methods}

We have computed atmospheric $P-T$ profiles and spectra for several planets with a plane-parallel model atmosphere code that has been used for a variety of planetary and substellar objects. The code was first used to generate profiles and spectra for Titan's atmosphere by McKay et al. (1989). It was significantly revised to model the atmospheres of brown dwarfs (Marley et al. 1996, 2002; Burrows et al. 1997) and irradiated giant planets (Marley \& McKay 1999, for Uranus). Recently, it has been applied to L- and T-type brown dwarfs (Saumon et al. 2006, 2007; Cushing et al. 2008) and hot Jupiters (Fortney et al. 2005, 2006b, 2007). It explicitly accounts for both incident radiation from the parent star and thermal radiation from the planet's atmosphere and interior. The radiative transfer solution algorithm was developed by Toon et al. (1989). We model the impinging stellar flux from 0.26 to $6.0 \mu \mathrm{m}$ and the emitted thermal flux from 0.26 to $325 \mu \mathrm{m}$.

We use the elemental abundance data of Lodders (2003) and chemical equilibrium compositions are computed with the CONDOR code, following Lodders \& Fegley $(2002,2006)$ and Lodders $(1999,2002)$. We maintain a large and constantly updated opacity database, which is described in Freedman et al. (2008). When including the opacity of clouds, such as Fe-metal and $\mathrm{Mg}$-silicates, we use the cloud model of Ackerman \& Marley (2001). However, in our past work we have found only weak effects on $P$ - $T$ profiles and spectra due to cloud opacity (Fortney et al. 2005), so we ignore cloud opacity here. However, the sequestering of elements into condensates, and their removal from the gas phase ("rainout") is always accounted for in the chemistry calculations. We note that day sides of the strongly irradiated $\mathrm{pM}$ class planets are too warm for Fe-metal and Mg-silicates condensates to form.

\subsection{Very Hot Jupiters and $\mathrm{TiO} / \mathrm{VO}$ Chemistry}

It is clear that the abundances to $\mathrm{TiO}$ and $\mathrm{VO}$ gases is important in these atmospheres. Hubeny et al. (2003) first discussed how understanding the "cold trap" phenomenon may be significant in understanding these abundances. If a given $P$ - $T$ profile crosses a condensation curve in two corresponding altitude levels, the condensed species is expected to eventually mix down to the highest pressure condensation point, where the cloud remains confined due to the planet's gravitational field. This process is responsible for the extremely low water abundance in the Earth's stratosphere. It can also be seen in the atmospheres Jupiter and Saturn, where the ammonia ice clouds are confined to a pressure of several bars, although both these planets exhibit stratospheres, such that their warm upper atmospheres pass the ammonia condensation curve again at millibar pressures. For the highly irradiated planets, the relevant condensates are those that remove gaseous $\mathrm{TiO}$ and $\mathrm{VO}$, and sequester $\mathrm{Ti}$ and $\mathrm{V}$ into solid condensates at pressures of tens to hundreds of bars, far below the visible atmosphere.

The cold trap phenomenon constitutes a departure from chemical equilibrium that cannot be easily accounted for in the pretabulated chemical equilibrium abundances used by Hubeny et al. (2003), Fortney et al. (2006b), Burrows et al. (2007b), and here as well. Our chemical abundances and opacities are pretabulated in $P$ - $T$ space and the atmosphere code interpolates in these abundances as it converges to a solution. The abundances determined for any one pressure level of the $P$ - $T$ profile are not

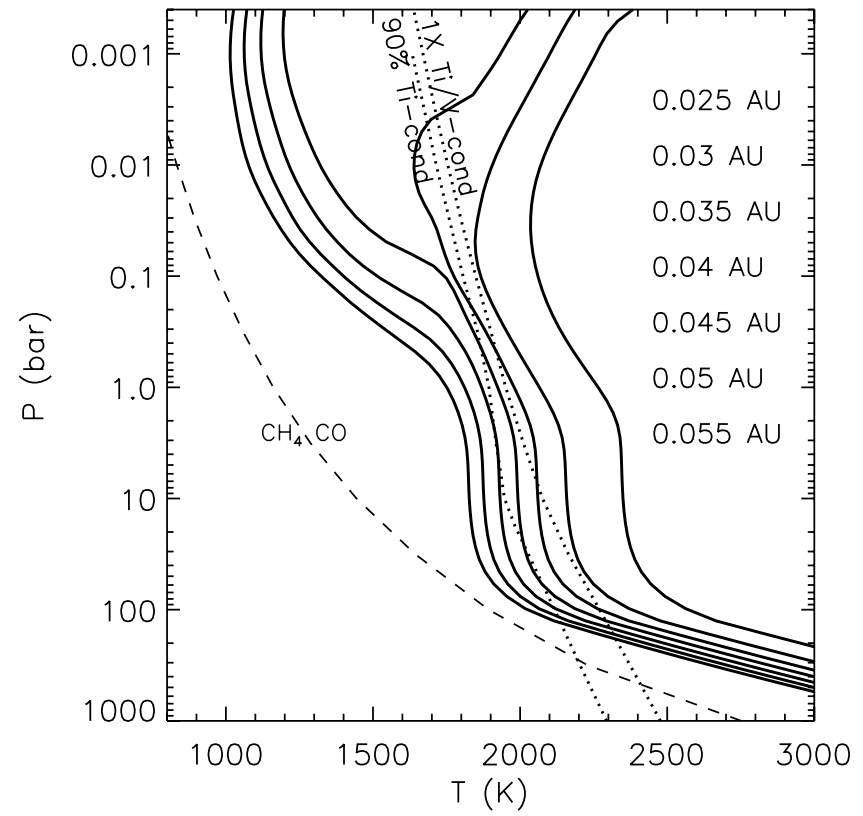

FIg. 2.-Model $P$ - $T$ profiles for planets with $g=15 \mathrm{~m} \mathrm{~s}^{-2}$ and $T_{\text {int }}=200 \mathrm{~K}$ at various distances $(0.025-0.055 \mathrm{AU})$ from the Sun. This $T_{\text {int }}$ value is roughly consistent with that expected for a $1 M_{\mathrm{J}}$ planet with a radius of $1.2 R_{\mathrm{J}}$. Condensation curves are dotted lines and the curve where $\mathrm{CO}$ and $\mathrm{CH}_{4}$ have equal abundances is dashed. The $0.1 \mathrm{X}$ Ti-Cond curve shows where $90 \%$ of the Ti has condensed out. However, even then $\mathrm{TiO}$ is a major opacity source. For none of these profiles has $\mathrm{TiO} / \mathrm{VO}$ been artificially removed. The kinks in the $0.035 \mathrm{AU}$ profile are due to interpolation difficulties, as the opacity drops significantly over a small temperature range.

cognizant of abundances of other levels of the profile, although condensation and settling of species is always properly accounted for. In this case a tabulated $\mathrm{TiO}$ abundance at a given $P-T$ point at which, in equilibrium, TiO would be in the gaseous state (warmer than required for Ti condensation), may not be correct. If the atmospheric $P$ - $T$ profile intersects the condensation curve, the atmosphere becomes depleted in TiO above the cloud. We do not treat the cold trap here. In practice, we use two different opacity databases: one with $\mathrm{TiO}$ and $\mathrm{VO}$ removed at $P<10$ bars, which simulates the removal of Ti and $\mathrm{V}$ into clouds, and one in which gases $\mathrm{TiO}$ and $\mathrm{VO}$ remain as calculated by equilibrium chemistry (which does not include depletion from a cold trap).

A full discussion of titanium and vanadium chemistry in the context of M- and L-dwarf atmospheres can be found in Lodders (2002). Much of that discussion pertains to the atmospheres of highly irradiated planets as well. The chemistry is complex. For instance Lodders \& Fegley (2006) (using the updated Lodders 2003 abundances) find that the first Ti condensate will not necessarily be $\mathrm{CaTiO}_{3}$. For solar metallicity, the first condensate is TiN if $P$ bars $\gtrsim 30$ bars, $\mathrm{Ca}_{3} \mathrm{Ti}_{2} \mathrm{O}_{7}$ if 5 bars $\lesssim P \lesssim 30$ bars, $\mathrm{Ca}_{4} \mathrm{Ti}_{3} \mathrm{O}_{10}$ if 0.03 bars $\lesssim P \lesssim 5$ bars, and $\mathrm{CaTiO}_{3}$ if $P \lesssim 0.03$ bars. These four condensates are the initial condensates as a function of total pressure and their condensation temperatures define the Ti-condensation curve in Figure 2. Another important point is that, following Lodders (2002), we assume that vanadium condenses into solid solution with Ti-bearing condensates, as is found in meteorites (Kornacki \& Fegley 1986). Lodders (2002) find this condensation sequence is also consistent with observed spectra at the M- to L-dwarf transition (Kirkpatrick et al. 1999). In the absence of this effect $V$ would not condense until $\sim 200 \mathrm{~K}$ cooler temperatures are reached and solid VO forms, as in the chemistry calculations of Burrows \& Sharp (1999), Allard 


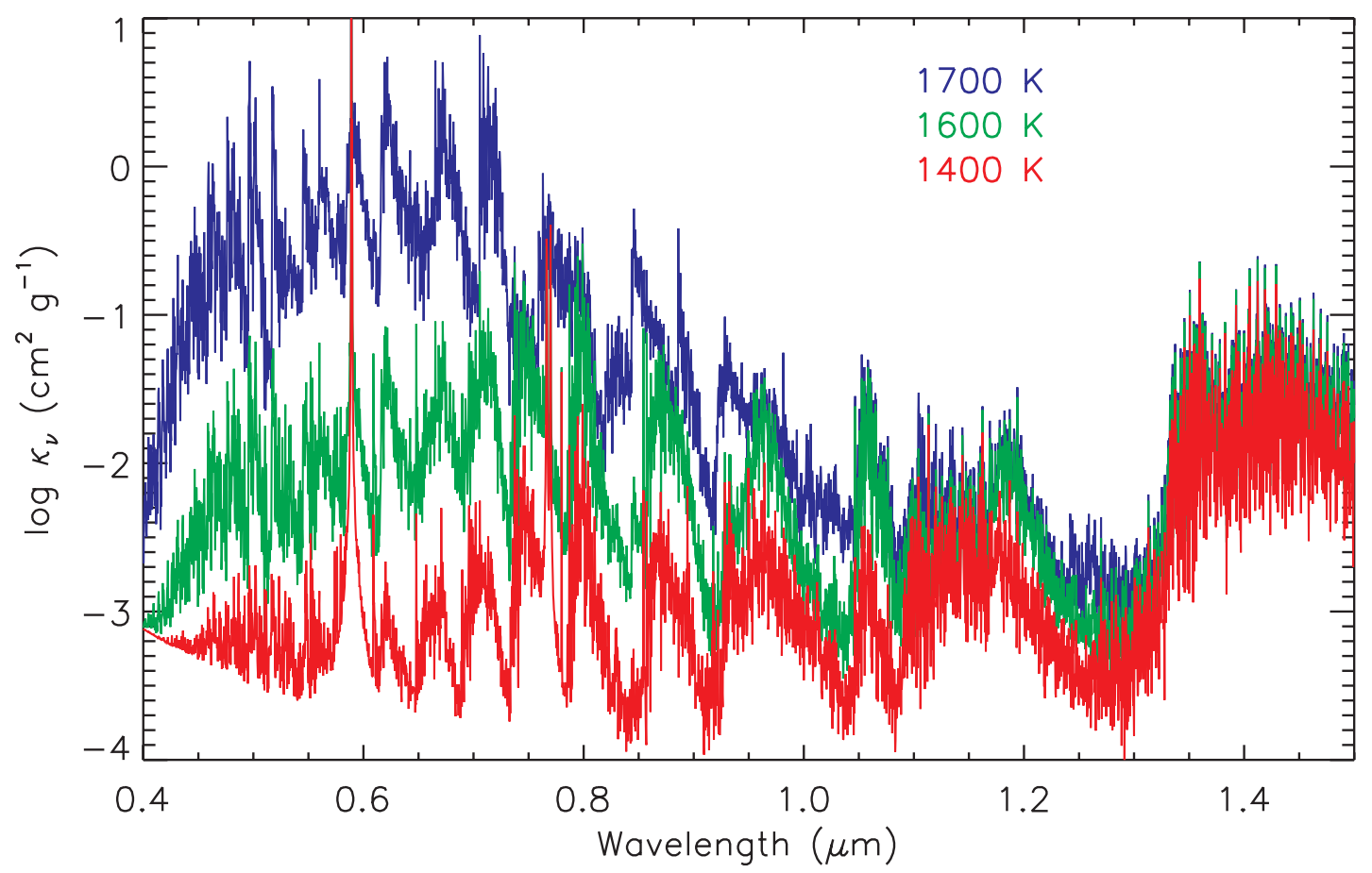

FIG. 3.- Total abundance-weighted atmospheric opacity at $P=1 \mathrm{mbar}$ and $T=1700$ (blue), 1600 (green), and 1400 (red) $\mathrm{K}$. The hottest temperature, $1700 \mathrm{~K}$, is warmer than the temperature for Ti and V condensation, which begins to occur at $1670 \mathrm{~K}$. The intermediate temperature, $1600 \mathrm{~K}$, is $33 \mathrm{~K}$ cooler than temperature at which $\mathrm{Ti}$ is $90 \%$ condensed. The optical opacity drops due to the removal of gaseous $\mathrm{TiO}$ and $\mathrm{VO}$ into Ti- and V-bearing solid condensates. By $1400 \mathrm{~K} \mathrm{TiO}$ and $\mathrm{VO}$ bands have given way to prominent water bands and alkali lines. A strong water vapor band at $1.4 \mu \mathrm{m}$ is readily seen at all three of these temperatures, and indicates the relatively modest change in absorption by water vapor with a $300 \mathrm{~K}$ drop in temperature.

et al. (2001), and Sharp \& Burrows (2007). Choices made in the calculation of the Ti condensation curve (e.g., number of potential Ti-bearing compounds included in the calculations) and the $\mathrm{V}$ condensation curve (e.g., whether $\mathrm{V}$ enters into Ti-bearing condensates or condenses as solid VO) will cause some shift in the position of these condensation curves, important boundaries between the $\mathrm{pL}$ and $\mathrm{pM}$ classes.

\subsection{Calculating Pressure-Temperature Profiles}

When modeling the atmospheres of irradiated objects with a one-dimensional, plane parallel atmosphere code it is necessary to weigh the stellar flux by a geometric factor if one is computing a profile for dayside average or planet-wide average conditions. Descriptions of this issue in solar system atmosphere modeling can be found in Appleby \& Hogan (1984) and McKay et al. (1989). In the context of highly irradiated EGPs, Burrows et al. (2004, 2006, 2007a), Barman et al. (2005), Seager et al. (2005), and Fortney \& Marley (2007) have all discussed this issue in some detail. All are approximations for atmospheric structures that are surely complex for these highly irradiated atmospheres. In this paper we make a straightforward choice to multiply the incident flux by a factor $f=0.5$ for a dayside average (since the atmosphere radiates over $2 \pi$ sr but intercepts flux over only $\pi$ radians) and assume normal incidence of flux, $\mu=1$. This differs slightly from choices in our previous papers (see Fortney \& Marley 2007). All profiles shown in this paper are relevant for the irradiated planetary day side.

In Figure 2 we show $P-T$ profiles as a function of distance from the Sun. At 0.025 and 0.03 AU the profiles are everywhere warmer that the condensation curve for a solar abundance of $\mathrm{Ti}$ and V. Essentially all $\mathrm{Ti}$ and $\mathrm{V}$ is expected to be in $\mathrm{TiO}$ and VO. By 0.035 AU, where the incident flux is weaker, the profile crosses this condensation curve, labeled " $1 \mathrm{X}$ Ti/V-Cond." To the left of this curve, the $\mathrm{TiO} / \mathrm{VO}$ abundances fall exponentially with decreasing temperature. To the right, the TiO/VO abundances are essentially constant. This particular $P-T$ profile is oddly shaped, since it samples a region of $P-T$ space in which the opacity drops rapidly with temperature. It crosses back to the right of the condensation curve, where it again finds the "full" abundance of TiO/VO. This is an example of a profile that violates the cold-trap (which we do not model), as at lower pressures, $\mathrm{TiO} / \mathrm{VO}$ abundances should be reduced, given the condensation of Ti and V below. The dotted curve labeled " $90 \%$ Ti-Cond" shows where $90 \%$ of Ti has been lost to a solid condensate (A. S. Lee \& K. Lodders 2008, in preparation). Figure 3 is a plot of optical and near-infrared opacity at 1 mbar and shows that $\mathrm{TiO} / \mathrm{VO}$ are still major optical opacity sources even after condensation has begun. At $1700 \mathrm{~K}$, before $\mathrm{Ti} / \mathrm{V}$ condensation, opacity [here $\log (\kappa)$ in $\mathrm{cm}^{2} \mathrm{~g}^{-1}$ ] in $V$-band is $\sim-0.5$. By $1600 \mathrm{~K}, 33 \mathrm{~K}$ cooler than the $90 \%$ Ti-Cond curve, this opacity is still an order of magnitude larger than the opacity at $1400 \mathrm{~K}$, by which point the strong $\mathrm{TiO} / \mathrm{VO}$ bands have given way to alkali lines and water bands.

That $\mathrm{TiO} / \mathrm{VO}$ gradually wane in importance is consistent with observations of dwarfs at the M-L transition (Lodders 2002). The mixing ratios of these species do not drop to zero at the solar abundances condensation curve. For model atmospheres more distant from the parent star (0.05 AU and beyond), the abundance of $\mathrm{TiO} / \mathrm{VO}$ continues to fall exponentially with temperature, such that in the upper atmosphere it is no longer a major opacity source.

We can examine how the derived $P-T$ profiles for the pM class planets vary as a function of $T_{\text {int }}$, a planet's intrinsic effective temperature in the absence of irradiation, and as a function of surface gravity. This is shown in Figure 4. Lower gravity planets have lower pressure photospheres and consequently have warmer 


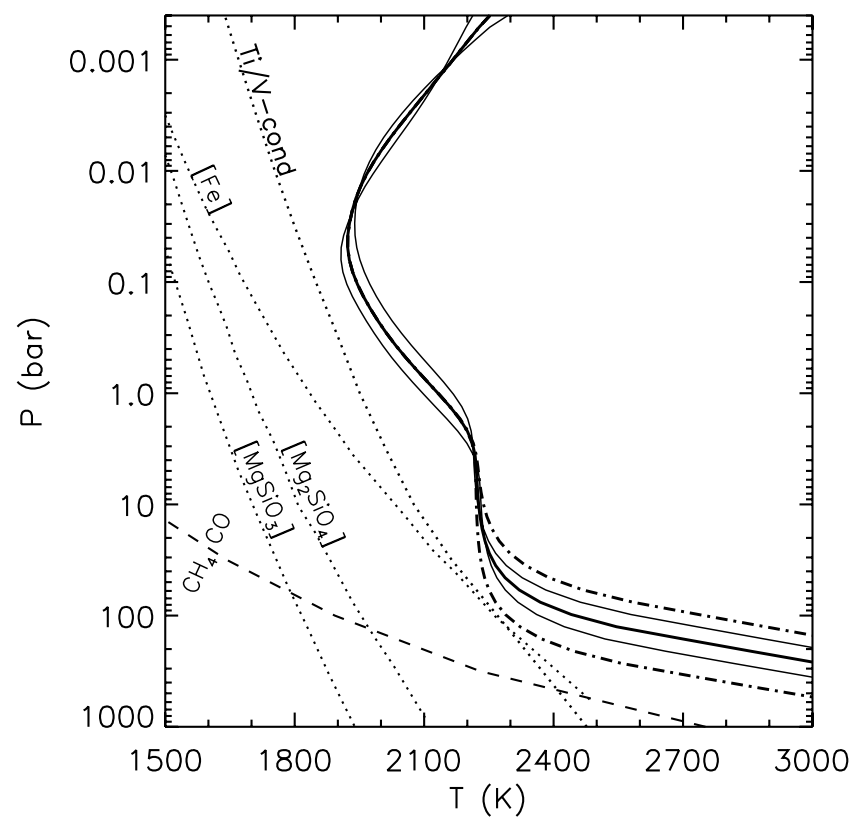

Fig. 4.- Model $P$ - $T$ profiles for planets at 0.028 AU from the Sun. Effects of different values $T_{\text {int }}$ and $g$ are shown. The thick solid profile is for $g=15 \mathrm{~m} \mathrm{~s}^{-2}$ and $T_{\text {int }}=200 \mathrm{~K}$. Lower and higher dash-dot profiles are for $g=15 \mathrm{~m} \mathrm{~s}^{-2}$ with $T_{\text {int }}$ values of 150 and $250 \mathrm{~K}$, respectively. Lower and higher thin solid profiles are for $T_{\text {int }}=200 \mathrm{~K}$ with $g$-values of 25 and $9 \mathrm{~m} \mathrm{~s}^{-2}$, respectively.

interiors, all else being equal, which has long been known from basic theory. As can be seen in Figure $1 b$, the lowest gravity planets cluster around $g=9 \mathrm{~m} \mathrm{~s}^{-2}$, while the highest gravity planets, with the exception of HD $147506 \mathrm{~b}$, cluster around $g=25 \mathrm{~m} \mathrm{~s}^{-2}$, similar to Jupiter. Both of these values are a factor of $\frac{2}{3}$ removed from our baseline $g=15 \mathrm{~m} \mathrm{~s}^{-2}$. The effect of gravity on the $P-T$ profiles for the pM class planets are relatively modest. However, the effect of $T_{\text {int }}$ is potentially important. We show profiles at $T_{\mathrm{int}}$ values of 150,200 , and $250 \mathrm{~K}$. Generally for adiabatic interiors, the higher the $T_{\text {int }}$, the warmer the interior, and the larger the radius of the planet. Independent of $T_{\text {int }}$, which could in principle be affected by barriers to convection (Stevenson 1985; Chabrier \& Baraffe 2007), the higher the entropy of the interior adiabat, the warmer the atmospheric adiabat, the larger the radius of the planet, and the less likely the deep atmosphere profile at 10 to 1000 bar will cross the Ti/V condensation boundary. Over gigayear timescales it may be possible for a planet's interior to cool enough such that significant $\mathrm{Ti} / \mathrm{V}$ condensation could occur at depth, and a planet could transition from $\mathrm{pM}$ to $\mathrm{pL}$ class.

In Figure 1 the gray shaded area is meant to illustrate a transition region between these classes of planets, where $\mathrm{TiO}$ and VO are waning in importance. Given the lack of data for the systems in this proposed transition region, the exact location and height of this region is merely suggestive. Analysis of the current secondary eclipse data for HD 189733b (Deming et al. 2006; Grillmair et al. 2007; Knutson et al. 2007) fits well with a pL class model (Fortney \& Marley 2007), and Knutson et al. (2007) directly measured a small day-night contrast, suggesting that $\mathrm{TiO} / \mathrm{VO}$ opacity has waned considerably at this incident flux level and there is no temperature inversion. We next turn to the upper boundary.

At the high incident fluxes encountered by HD 149026b, the temperature inversion predicted by Fortney et al. (2006b) neatly explains the large planet-to-star flux ratio at $8 \mu \mathrm{m}$ (Harrington et al. 2007). At lower incident fluxes, the large day-night tem- perature contrasts implied for $v$ And $\mathrm{b}$ and HD 179949 imply temperature inversions as we will show in $\S 4.2$ (and pM classification) at this flux level. Intriguingly, at a still lower irradiation level, Burrows et al. (2007b) find that a strong temperature inversion is needed for HD 209458b, an issue we also address in $\S 5.1$, which indicates that inversions extend down to at least this incident flux level. This inversion persists even though it is clear from Figure 2 that Ti/V condensation has begun in our one-dimensional model at this irradiation level (although is has not for the more irradiated $v$ And $\mathrm{b}$, as we show in $\S 5.2$ ). Similar to the findings of Burrows et al. (2007b), we can only obtain a temperature inversion for HD $209458 \mathrm{~b}$ induced by $\mathrm{TiO} / \mathrm{VO}$ opacity if their abundances are not diminished by the simple cold trap description discussed above. In $\S \S 5.1$ and 7.1 we discuss a few avenues to toward understanding if $\mathrm{TiO} / \mathrm{VO}$ remains the prominent stratospheric absorber down to this incident flux level.

The transition area as plotted in Figure 1 is thus given from mix of the observations and theory available to date. Additional complications will be effects of atmospheric metallicity on Ti/V condensation, the treatment of the incident stellar flux, planet surface gravity, and the temperature of the interior adiabat. For instance, it is certainly possible that a planet could appear marginally on to the hot side of this divide, but with a very cool interior (due to low mass and/or old age), it would have Ti/V condensed such that it is a $\mathrm{pL}$ class member. With better knowledge of the incident flux deposition and $\mathrm{Ti} / \mathrm{V}$ condensation, it may eventually be possible to "take the temperature of the interior adiabat" at pressures of 10 to 100 bars, which could shed light on the interior structure of some of these planets.

\section{RESULTS AND PREDICTIONS}

\subsection{Absorption and Emission of Flux}

It is worthwhile examining in some detail how the atmospheres of the $\mathrm{pM}$ class and $\mathrm{pL}$ class planets differ in the absorption of stellar flux as a function of atmospheric pressure. One can plot the layer net fluxes for several pressures as a function of wavelength to illustrate the main atomic and molecular absorbers in the atmosphere. Since these models are in radiative equilibrium, the integrated absorbed flux within a layer should equal the integrated emitted flux from that same layer. We can plot the net and integrated thermal flux to examine at what wavelengths, and which species, lead to atmospheric heating and cooling. A series of plots are shown in Figures 5 and 6, for a pL class planet at $0.05 \mathrm{AU}$, and Figures 7 and 8 , for a pM class planet at $0.03 \mathrm{AU}$. The left ordinate of these plots shows the quantity in ergs s${ }^{-1} \mathrm{~g}^{-1} \mu \mathrm{m}^{-1}$, which is the power per wavelength interval, per gram of atmosphere, absorbed or emitted in a slab of atmosphere, although we maintain the term "flux" for convenience.

In Figure 5 five panels are shown, from $0.45 \mathrm{mbar}$ to $4.1 \mathrm{bar}$, each separated by approximately an order of magnitude in pressure. Prominent in the top two panels are absorption of incident flux by neutral atomic alkalis $\mathrm{Na}($ at $0.59 \mu \mathrm{m}$ ) and $\mathrm{K}$ (at $0.77 \mu \mathrm{m}$ ) as well as strong $\mathrm{H}_{2} \mathrm{O}$ bands in the near-infrared. While $\mathrm{Na}$ and $\mathrm{K}$ lead to strong absorption in optical wavelengths, causing extremely dark atmospheres in the optical (Sudarsky et al. 2000; Seager et al. 2000), at 0.45 and 4.4 mbar, respectively, 4 and 1.5 times more incident stellar is actually absorbed by $\mathrm{H}_{2} \mathrm{O}$ than by alkalis. The third panel, $43 \mathrm{mbar}$, shows that essentially all incident flux at the wavelengths of the $\mathrm{Na}$ and $\mathrm{K}$ line cores have been absorbed, and the alkali line wings are now as important as the $\mathrm{H}_{2} \mathrm{O}$ bands in absorption. By 420 mbar, the wavelengths 


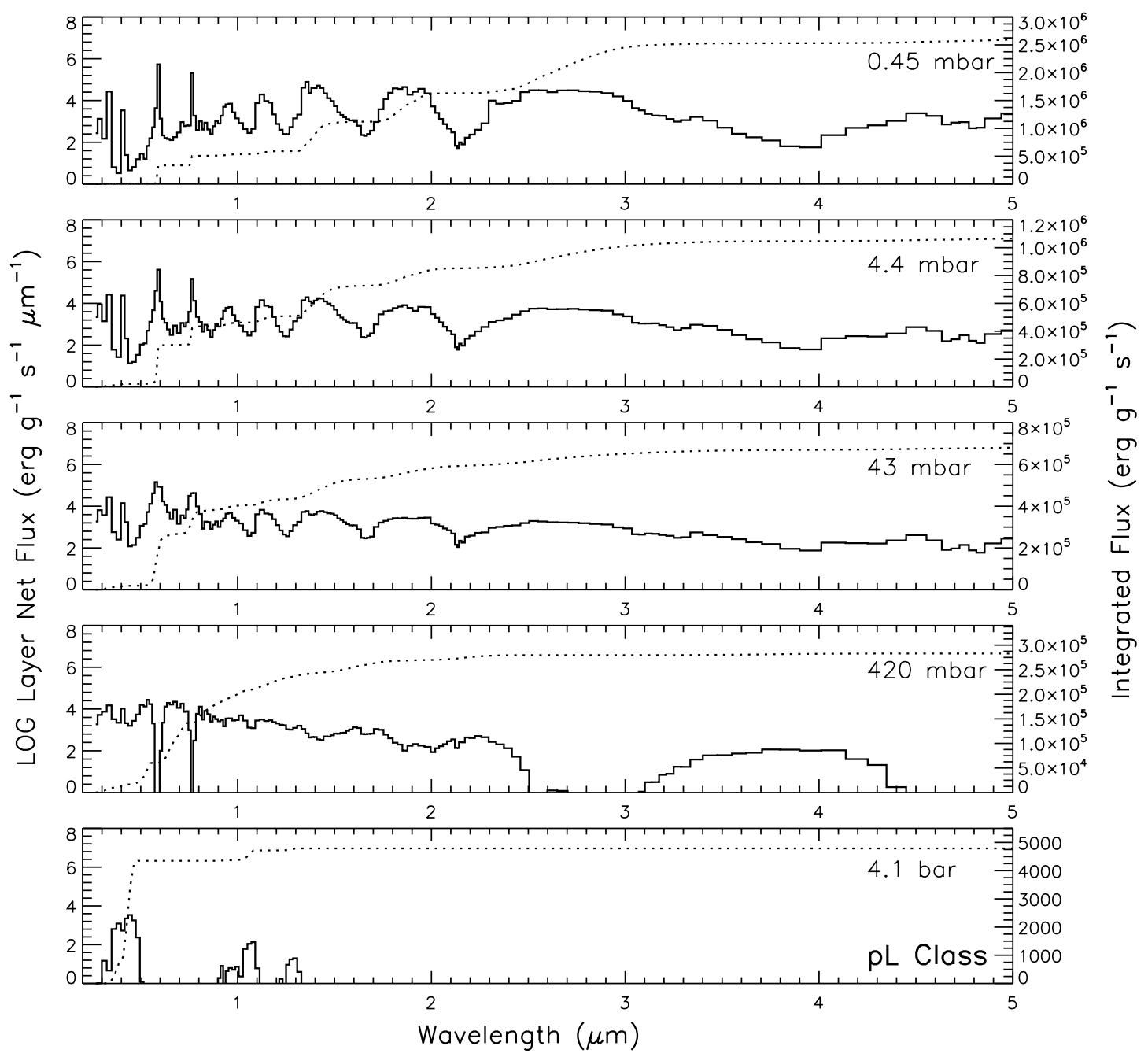

Fig. 5. - Net incident "flux" (ergs $\mathrm{g}^{-1} \mathrm{~s}^{-1} \mu \mathrm{m}^{-1}$; solid line, left ordinate) absorbed in five model layers for a cloud-free $\mathrm{pL}$ class model with TiO/VO removed, $g=15 \mathrm{~m} \mathrm{~s}{ }^{-2}$, at $0.05 \mathrm{AU}$ from the Sun. The dotted line (right ordinate) illustrates the running integrated flux, evaluated from short to long wavelengths. The layer integrated flux is read at the intersection of the dotted line and the right ordinate. Note the logarithmic scale on the left. At $0.45 \mathrm{mbar}$, although the absorption due to neutral atomic alkalis are important, more flux is absorbed by water vapor. Heating due to alkali absorption becomes relatively more important as pressure increases. By $420 \mathrm{mbar}$, there is no flux left in the alkali line cores, and by 4.1 bar, nearly all the incident stellar flux has been absorbed. (After Marley \& McKay 1999.)

corresponding to the broad $\mathrm{Na}$ and $\mathrm{K}$ lines have already become optically thick, leaving little flux left. About $\sim 10^{3}$ less flux is absorbed at 4.1 bar than $0.45 \mathrm{mbar}$. The pressure is now in the nearly isothermal region of the $P$ - $T$ profile, as shown in Figure 2. In summary, $\mathrm{Na}, \mathrm{K}$, and $\mathrm{H}_{2} \mathrm{O}$ are the major absorbers and heating in the millibar region of the atmosphere amounts to $\sim 10^{6} \mathrm{ergs} \mathrm{g}^{-1} \mathrm{~s}^{-1}$.

We can examine Figure 6 to understand the cooling of the atmosphere as these same pressure levels. This figure has a much larger wavelength range that is shown on a log scale. In the top three panels, from 0.45 to $43 \mathrm{mbar}$, it is predominantly the nearand mid-infrared water bands that allow the atmosphere to cool to space. Between $\sim 0.8-1.8 \mu \mathrm{m}$, positive layer net fluxes occur due to absorption of flux from above, by $\mathrm{H}_{2} \mathrm{O}$. Deeper in the atmosphere, at higher temperatures, the local Planck function at a given layer overlaps shorter wavelength water bands from $\sim 1$ to $2 \mu \mathrm{m}$ that are primarily responsible for cooling.

In Figure 7 we can clearly see how this pM class planet differs from the $\mathrm{pL}$ class. The incident stellar flux is higher and more of this flux is deposited into the 0.45 mbar layer of this model, as compared to that shown in Figure 5. This deposition occurs almost entirely through absorption by $\mathrm{TiO}$ and $\mathrm{VO}$ bands in the optical and near-infrared. Water vapor still absorbs flux, but its effect is swamped by the $\mathrm{TiO} / \mathrm{VO}$ absorption. This continues at $4.4 \mathrm{mbar}$, but already by $43 \mathrm{mbar}$, the vast majority of the incident flux has been absorbed, especially in the optical wavelengths. By 420 mbar there is little flux left in the optical and by 4.1 bar there is little flux left at any wavelength; again this pressure is in the nearly isothermal region shown in Figure 2.

The top panel of Figure 8 shows, since the atmosphere has absorbed a significant amount of flux at low pressures, compared to the $\mathrm{pL}$ class model, that the atmosphere must reach a much higher temperature to be able to adequately radiate away this energy. The local Planck function moves significantly blueward, so that it can radiate by means of the strong $\mathrm{H}_{2} \mathrm{O}$ near-infrared bands as well as the optical bands of TiO and VO. At 4.4 and 43 mbar, the atmosphere is cooler, and radiation by $\mathrm{H}_{2} \mathrm{O}$ bands is more important. At 43 mbar some heating from above does occur via $\mathrm{TiO} / \mathrm{VO}$ absorption. At $420 \mathrm{mbar}$ and $4.1 \mathrm{bar}$, the local temperature has again increased, leading to radiation again most prominently by the near-infrared $\mathrm{H}_{2} \mathrm{O}$ bands.

\subsection{Radiative Time Constants}

It is clear that a key difference between the atmospheres of the $\mathrm{pL}$ class planets and $\mathrm{pM}$ class planets is the pressures at which the absorption and emission of flux occurs. This can be shown in 


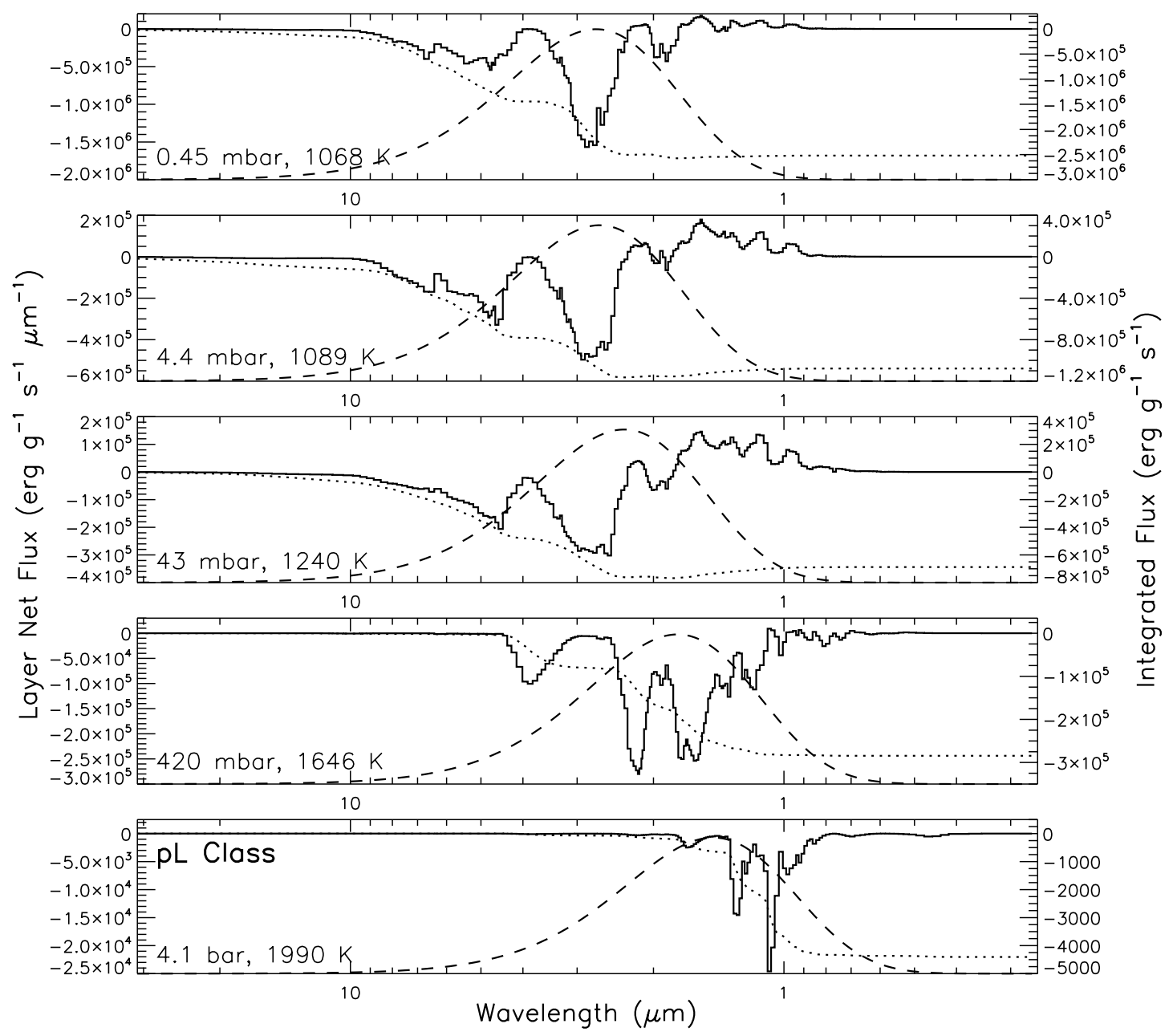

FIG. 6.- Similar to Fig. 5, but for thermal emission, from 30 to $0.26 \mu \mathrm{m}$, from long wavelengths to short. Negative flux is emitted. The dotted line is again integrated flux, evaluated from long to short wavelengths. The negative value of a layer integrated flux in Fig. 5 equals the integrated flux here. In addition, the scaled Planck function appropriate for each layer is shown as a dashed curve. The temperature and pressure of each layer is labeled. Cooling occurs mostly by way of water vapor, but also CO. As the atmosphere cools with altitude progressively longer wavelength water bands dominate the layer thermal emission.

a more straightforward manner by means of the brightness temperature, $T_{B}$. One can then examine the $P-T$ profile to find the pressure that corresponds to a given $T_{B}$, which is the characteristic atmospheric pressure for this thermal emission. This quantity is plotted as a function of wavelength in Figure 9. In general thermal emission arises from a pressure level roughly an order of magnitude greater in a $\mathrm{pL}$ class atmosphere than in a pM class atmosphere, due to the higher opacity in the hotter pM atmospheres, both at optical wavelengths, due to $\mathrm{TiO} / \mathrm{VO}$, and infrared wavelengths, due to increased $\mathrm{H}_{2} \mathrm{O}$ opacity and $\mathrm{H}_{2}$ collision induced absorption.

What effect this may have on the dynamical redistribution of energy in a planetary atmosphere can be considered after calculation of the radiative time constant, $\tau_{\text {rad }}$. In the Newtonian cooling approximation a temperature disturbance relaxes exponentially toward radiative equilibrium with a characteristic time constant $\tau_{\text {rad }}$ (e.g., Goody \& Yung 1989; Salby 1996). At photospheric pressures this value can be approximated by

$$
\tau_{\text {rad }} \sim \frac{P}{g} \frac{c_{P}}{4 \sigma T^{3}},
$$

where $\sigma$ is the Stefan-Boltzmann constant and $c_{P}$ is the specific heat capacity (Showman \& Guillot 2002). However, this quan- tity can be derived anywhere in the radiative atmosphere via the following formulation. We first obtain a $P-T$ profile solution that is in radiative-convective equilibrium. We can then take this profile, and in a given layer include a small $(\sim 10 \mathrm{~K})$ thermal perturbation, $\Delta T$. With this $\Delta T$ in place we perform a radiative transfer calculation, and additionally calculate the flux divergence $d F / d z$, which is zero in radiative equilibrium (where $z$ is the height). For a function $f(t)$ a time constant will be given by $f(d f / d t)^{-1}$. We can then solve for $\tau_{\text {rad }}$ as

$$
\tau_{\mathrm{rad}}=\Delta T \frac{\rho c_{P}}{d F / d z}
$$

where $\rho$ is the mass density. The quantity $(d F / d z) /\left(\rho c_{P}\right)$ is the heating/cooling rate in $\mathrm{K} \mathrm{s}^{-1}$. By varying the location of the $\Delta T$ perturbation with height, one can calculate $\tau_{\text {rad }}$ as a function of pressure in the atmosphere, for a given $P-T$ profile. In practice we find values of $\tau_{\text {rad }}$ that are very similar to those derived by Iro et al. (2005) for HD 209458b. Interestingly, these authors performed a much different calculation that involved the input of Gaussian temperature fluctuations and the direct calculation of the time necessary for their code to relax back to radiative equilibrium. A larger exploration of $\tau_{\text {rad }}$ over a wider range of phase space, with additional discussion, will be found in A. P. Showman et al. (2008, in preparation). 


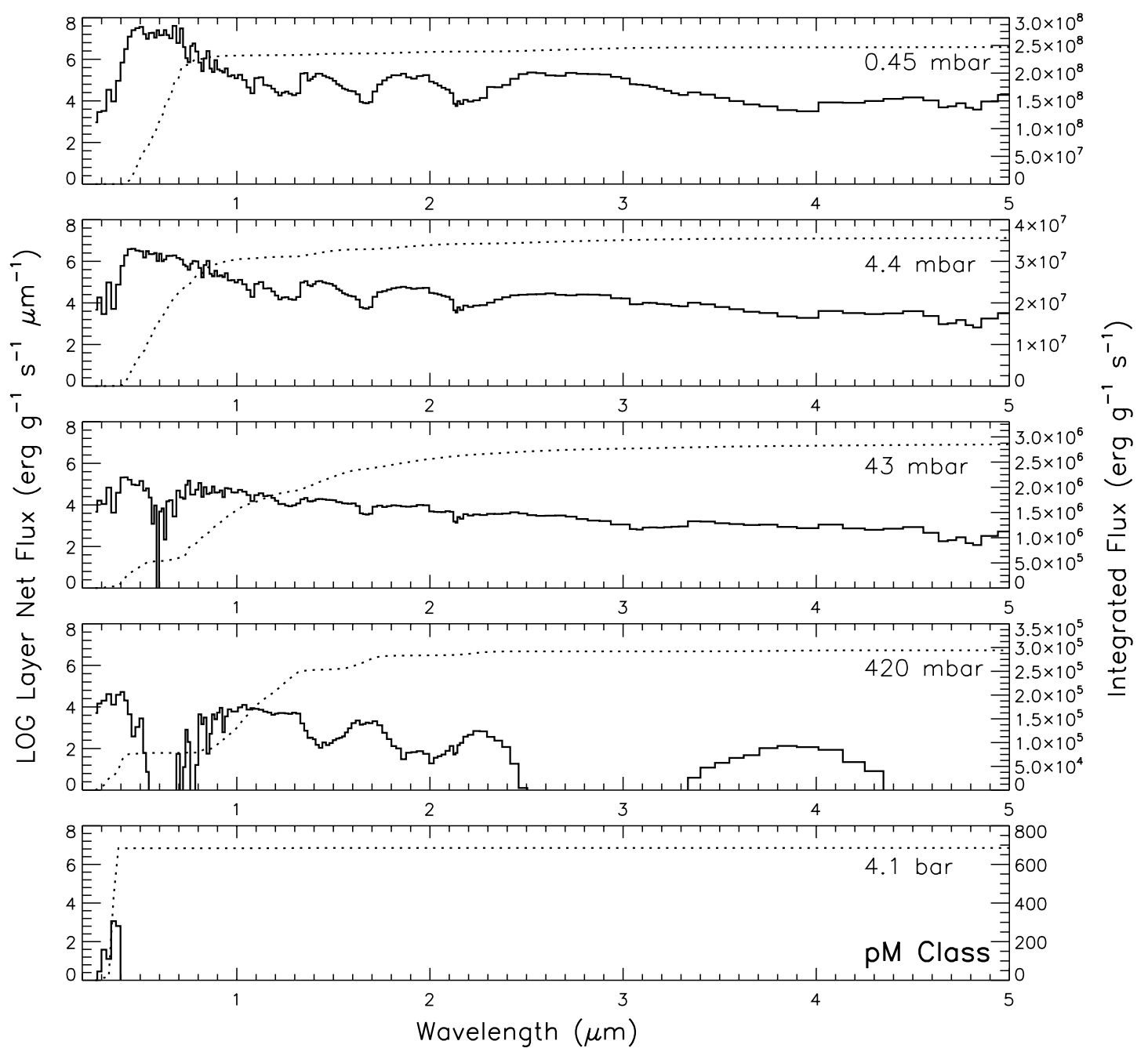

FIG. 7.- Net incident "flux" (ergs $\mathrm{g}^{-1} \mathrm{~s}^{-1} \mu \mathrm{m}^{-1}$, solid line, left ordinate) absorbed in five model layers for a pM class model with $g=15 \mathrm{~m} \mathrm{~s}$ at 0.03 AU from the Sun. Only the left ordinate scale is the same as in Fig. 5. This pM class model possesses a hot stratosphere. At 0.45 and 4.4 mbar absorption of incident flux (heating) is due almost entirely to optical bands of $\mathrm{TiO}$ and $\mathrm{VO}$. Water vapor also absorbs flux as in the pL class model, but its effect is comparatively swamped. Also similar to the model shown in Fig. 5, by 420 mbar most flux at optical wavelengths has already been absorbed.

In Figure 10 we show our calculated $\tau_{\text {rad }}$ as a function of pressure alongside the radiative-convective equilibrium profiles from which they were generated. Although these time constants are nearly equivalent in the dense lower atmosphere, they are significantly different in the thinner upper atmosphere, which one senses from mid-infrared observations.

The right $y$-axis in Figure 9 is cast in terms of the radiative time constant appropriate for a given pressure in the atmosphere (e.g., Seager et al. 2005). Note that this axis is not linear: each major tick mark is labeled with the $\tau_{\text {rad }}$ that is appropriate for the major tick mark at the left. While those for the pM class range from only $10^{3}$ to $10^{4} \mathrm{~s}$, those for the $\mathrm{pL}$ class range from $10^{4}$ to $10^{5} \mathrm{~s}$. This $\sim 10$ times difference in timescale is a consequence of both the hotter temperatures and lower pressures of the $\mathrm{pM}$ class photospheres, as can be understood from equation (1).

One can also define an advective timescale, a characteristic time for moving atmospheric gas a given planetary distance. A common definition is

$$
\tau_{\text {advec }}=\frac{R_{p}}{U},
$$

where $U$ is the wind speed and $R_{p}$ is the planet radius (Showman $\&$ Guillot 2002; Seager et al. 2005). If one sets $\tau_{\text {advec }}=\tau_{\text {rad }}$ and sets $R_{p}=1 R_{\mathrm{J}}$, one can derive the wind speed $U$ that would be necessary to advect atmospheric gas before a $\tau_{\text {rad }}$ has elapsed. This is also shown in Figure 9, via the gray bars on the right side. Since depth to which one "sees" is wavelength dependent, the apparent efficiency of atmospheric dynamics in redistributing energy in the planet's atmosphere will depend on the wavelength of observations. For wavelengths at which the atmospheric opacity is low, one sees flux emerging from relatively deep layers. The planet will then appear more homogenized (Seager et al. 2005), as was shown explicitly by Fortney et al. (2006a) for light curves for the Cooper \& Showman (2006) three-dimensional dynamical model. Therefore, the shape and times of maxima and minima in thermal emission light curves will be a function of wavelength.

Examination of Figure 9 shows that for a $\mathrm{pM}$ class planet wind speeds of dozens of kilometers per second would be necessary for advection to dominate over cooling/heating! For a pL class planet more modest winds speeds of several kilometers per second are needed. Several kilometer per second winds, which are similar to the sound speed, are in line with predictions of a number of two-dimensional and three-dimensional dynamical models for hot Jupiter atmospheres (Showman \& Guillot 2002; Cooper \& Showman 2005; Langton \& Laughlin 2007; DobbsDixon \& Lin 2008). These calculated wind speeds are far below 


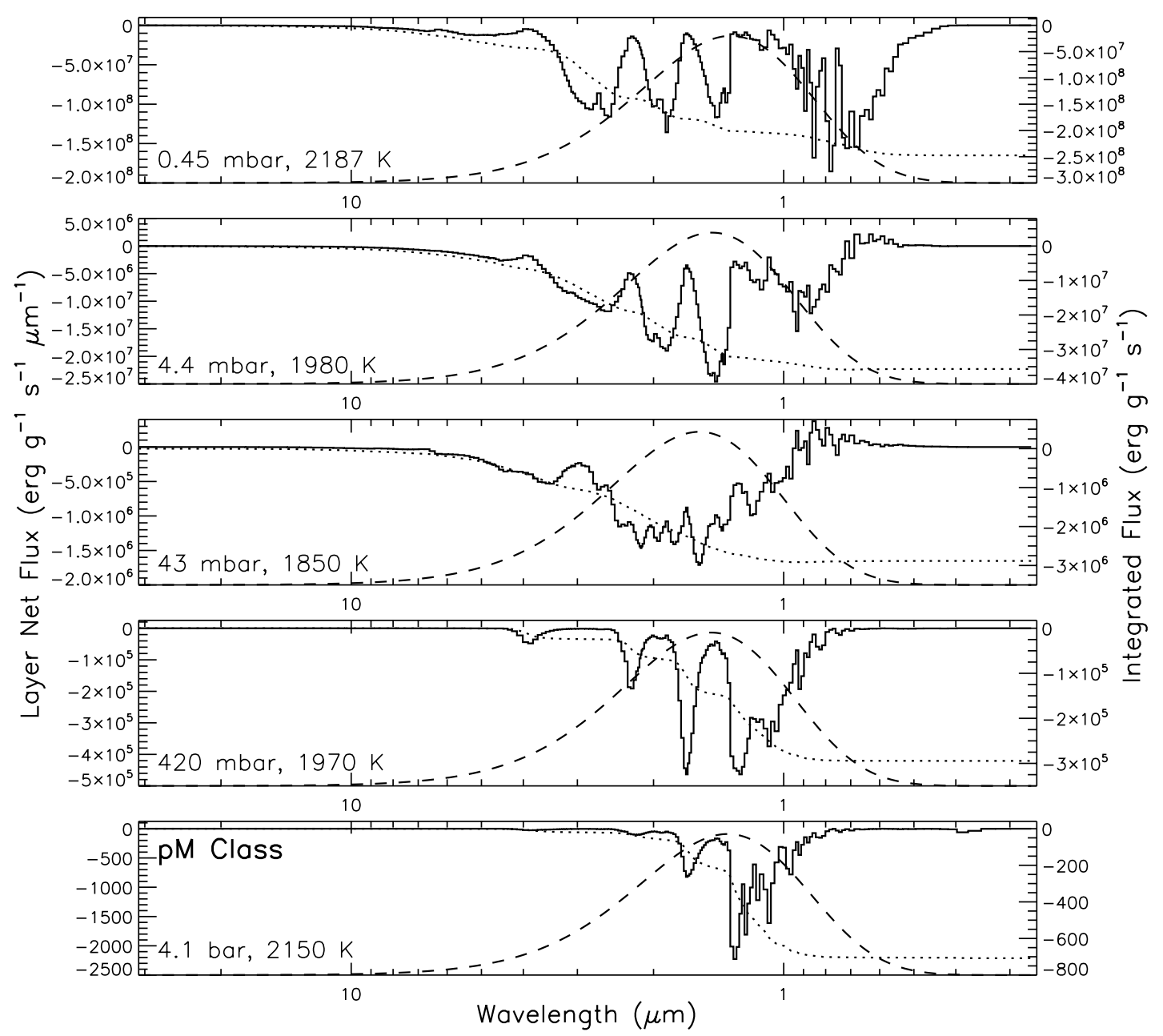

Fig. 8. - Again the pM class model, as shown in Fig. 7, but now for thermal emission. At the topmost layer shown, the local Plank function must move to high temperatures to effectively radiate by means of bands of water, $\mathrm{TiO}$, and $\mathrm{VO}$. At higher pressure layers, there is back-heating due to $\mathrm{TiO}$ and $\mathrm{VO}$ emission from above (seen especially at 4.4 and 43 mbar), along with cooling by water vapor.

those needed to advect air before it radiatively heats/cools in a pM class atmosphere.

Winds will not be able to redistribute energy at the photospheres of pM class planets. The atmospheres of pM class planets likely appear as one would expect from radiative equilibrium considerations: the hottest part of the atmosphere is at the substellar point and the atmosphere is cooler as one moves toward the planet's limb. Deviations from this prediction may occur from 1-2 $\mu \mathrm{m}$ where opacity windows allow for observation of flux from relatively higher pressures. The night-side temperature will be relatively cold and will be set by the efficiency of energy redistribution at depth, as well as the intrinsic flux from the interior of the planet. In the absence of a large intrinsic flux driven by an interior energy source, such as tides, redistribution may well swamp the intrinsic flux. For a pL class atmosphere, there will be a much more complex interplay between radiation and dynamics; energy redistribution will more readily lead to a planetary hot spot being blown downwind. The location of the hot spot will itself be wavelength dependent, and there will increased energy transfer to the night side at photospheric pressures. Recently, Dobbs-Dixon \& Lin (2008) have shown that larger day-night temperature differences are expected with increased atmospheric opacity, but their models lack motivation for their opacity choices. Dynamical models that do not include a realistic treatment of opacities and radiative transfer will miss the important differences between these two classes of planets.

\subsection{Characterization via Transit Observations}

Primary transit observations for $\mathrm{pL}$ class planets HD 209458b and HD 189733b have yielded detections of sodium (Charbonneau et al. 2002) and water vapor (Barman 2007; Tinetti et al. 2007). Since water vapor is present in abundance in $\mathrm{pM}$ class planets, it should be detectable as well. Observations in the optical would show strong absorption due to $\mathrm{TiO}$ and $\mathrm{VO}$ across a broad wavelength range, which will lead to observed transit radii (Hubbard et al. 2001; Baraffe et al. 2003; Burrows et al. 2003) that correspond to even lower atmospheric pressure (higher in the atmosphere) that those for $\mathrm{pL}$ class planets.

We perform a simple calculation to illustrate this point. From Figure 9 the approximate pressure levels for thermal emission are known. After calculating the distances between the wavelength-dependent photospheric pressures to a reference pressure of 1 bar, we can follow the implementation of Burrows et al. (2007a) to derive the (even larger) radius at which the slant optical depth reaches unity. This is the radius one measures (at a given wavelength or wave band) during a transit observation. From Burrows et al. (2007a) the extension $d R$ from the photospheric radius to the transit radius is approximately

$$
d R=H \ln \sqrt{\frac{2 \pi R_{p}}{H}}
$$




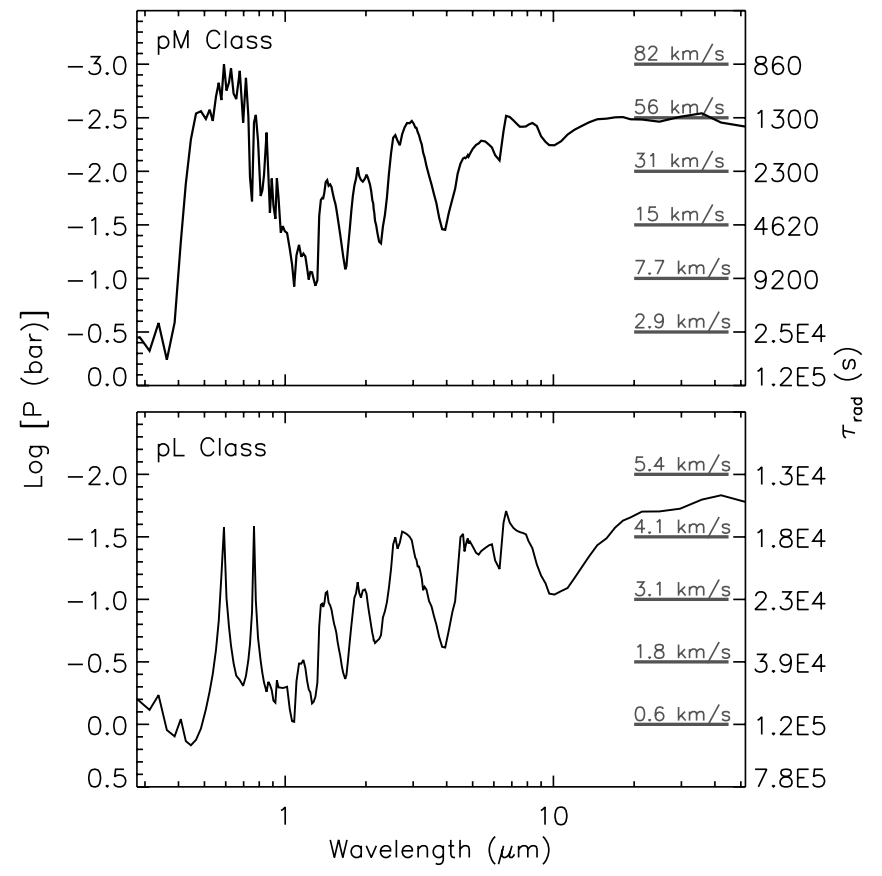

FIG. 9.- Both panels show, as a function of wavelength, the atmospheric pressure that corresponds to a given brightness temperature. Note the differences in the $y$-axes. Top: Planet at $0.03 \mathrm{AU}$ that has a hot stratosphere induced by absorption by TiO/VO. Bottom: Planet at $0.05 \mathrm{AU}$ that lacks a temperature inversion. The right ordinate shows the corresponding radiative time constant at each major tick mark from the pressure axis. Note that this right axis is not linear. The labeled gray lines at right indicate an advective wind speed that would be necessary to give an advective timescale equal to the given radiative constant see text). For instance, in the top panel, at $\log P=-2.0$, the radiative time constant is $2300 \mathrm{~s}$, and an advection time of $2300 \mathrm{~s}$ would require a wind speed of $31 \mathrm{~km} \mathrm{~s}^{-1}$.

where $H$ is the characteristic atmospheric scale height and $R_{p}$ is the planet radius. We plot the approximate transit radius for the model pL class planet and pM class planet in Figure 11. Here the 1 bar radius is $1.20 R_{\mathrm{J}}$ and the characteristic $T$-values for the scale height calculation for the $\mathrm{pL}$ and $\mathrm{pM}$ class planets are 1070

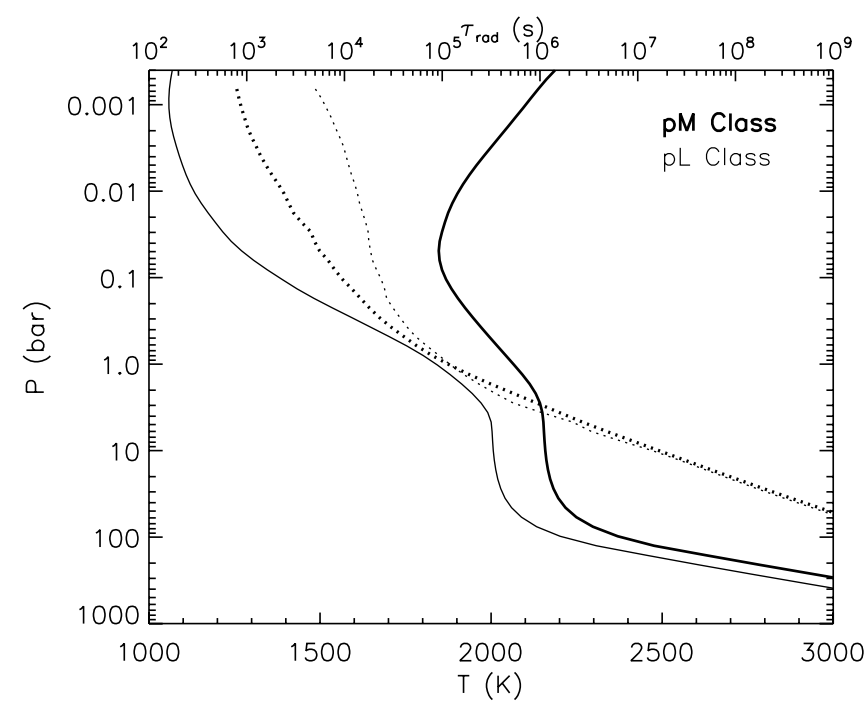

FIG. 10.-Pressure-temperature (solid lines, bottom $x$-axis) and pressure- $\tau_{\text {rad }}$ profiles (dotted lines, top $x$-axis) for the models at $0.03 \mathrm{AU}$ ( $\mathrm{pM}$ class, thick lines) and $0.05 \mathrm{AU}$ ( $\mathrm{pL}$ class, thin lines, with $\mathrm{TiO} / \mathrm{VO}$ removed). $\mathrm{TiO} / \mathrm{VO}$ has been removed when calculating the $0.05 \mathrm{AU}$ profile. Comparing this profile to the one at $0.05 \mathrm{AU}$ in Fig. 2, where $\mathrm{TiO} / \mathrm{VO}$ were not removed, shows slight differences around 1 bar, where equilibrium chemistry predicts a local increase in the $\mathrm{TiO} / \mathrm{VO}$ abundances.

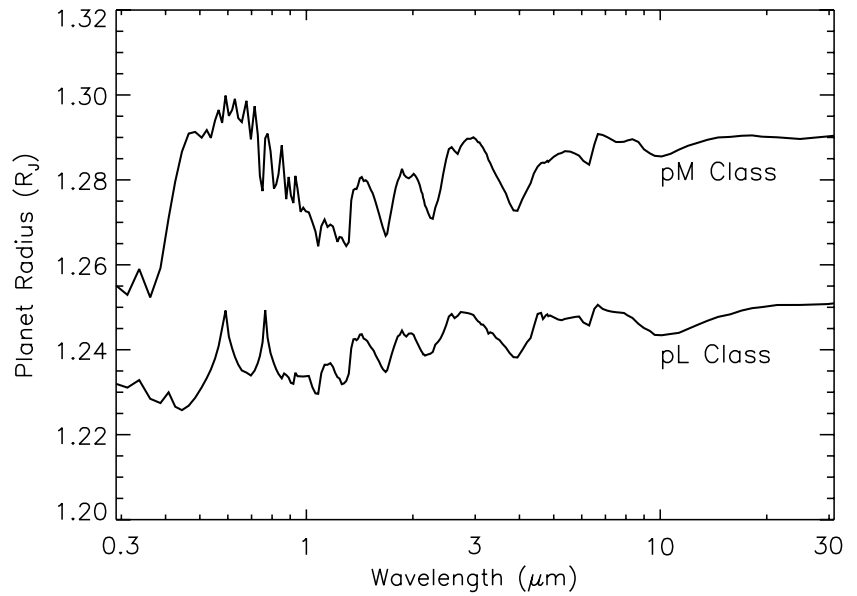

FIG. 11.-Approximate radius one would observe as a function of wavelength for a pL class and a pM class planet with a 1 bar radius of $1.20 R_{\mathrm{J}}$ and $g=15 \mathrm{~m} \mathrm{~s}^{-1}$. (See text for discussion.)

and $2000 \mathrm{~K}$, respectively. Since our derived $P-T$ profiles do not reach to microbar pressures, a more detailed transit radius calculation using the precise run of temperature and opacity versus pressure (see Hubbard et al. 2001; Fortney et al. 2003) will be investigated in future work. Nonetheless, the important points are clear. Due predominantly to the hotter atmosphere of $\mathrm{pM}$ class planets, their atmospheric extension is larger. In addition, as suggested by Burrows et al. (2007b) the strong opacity of TiO and $\mathrm{VO}$ leads to a larger radius in a wide optical band, compared to $\mathrm{pL}$ class planets. Figure 11 shows a difference of $\sim 5 \%$ at optical wavelengths. This difference in radius could be even further enhanced by temperatures in excess of the $2000 \mathrm{~K}$ used in the calculation. This plot should only be considered suggestive, as a more detailed calculation would involve different chemical abundances on the day and night side of the planetary limb. Cooler limb temperatures may not allow for gaseous $\mathrm{TiO}$ and $\mathrm{VO}$.

Detailed secondary eclipse observations may shed additional light on the structure of these atmospheres. As was shown in Fortney et al. (2006b) for HD 149026b, planets with temperature inversions will show limb brightening, rather than darkening, which may eventually be detectable via secondary eclipse mapping (Williams et al. 2006; Rauscher et al. 2007b).

\section{APPLICATION TO KNOWN PLANETS}

\subsection{HD $209458 b$ at Secondary Eclipse}

Recently, Knutson et al. (2008) published observations of the secondary eclipse of planet HD 209458b. These observations are from Spitzer IRAC, and they constitute the first published observations for a transiting planet across all four IRAC bands. These observed planet-to-star flux ratios were interpreted as being caused by a temperature inversion (hot stratosphere) on the day side of the planet (Burrows et al. 2007b; Knutson et al. 2008). The reasons for this interpretation include large brightness temperatures relative to its expected day-side $T_{\text {eff }}$, especially at 4.5 and $5.8 \mu \mathrm{m}$, and a large flux ratio at $4.5 \mu \mathrm{m}$ (relative to $3.6 \mu \mathrm{m}$ ), which is enhanced due to strong $\mathrm{CO}$ and $\mathrm{H}_{2} \mathrm{O}$ emission features from 4 to $6 \mu \mathrm{m}$ (Fortney et al. 2006b; Burrows et al. 2007b). In our terminology, HD 209458b joined HD $149026 b$ as a pM class planet.

In Figure 12 (left) we plot $P$-T profiles for HD 209458b, with and without the opacity of $\mathrm{TiO}$ and VO. As was previously shown (Hubeny et al. 2003; Fortney et al. 2006b; Burrows et al. $2007 b$ ), absorption of stellar flux by $\mathrm{TiO} / \mathrm{VO}$ leads to a hotter 

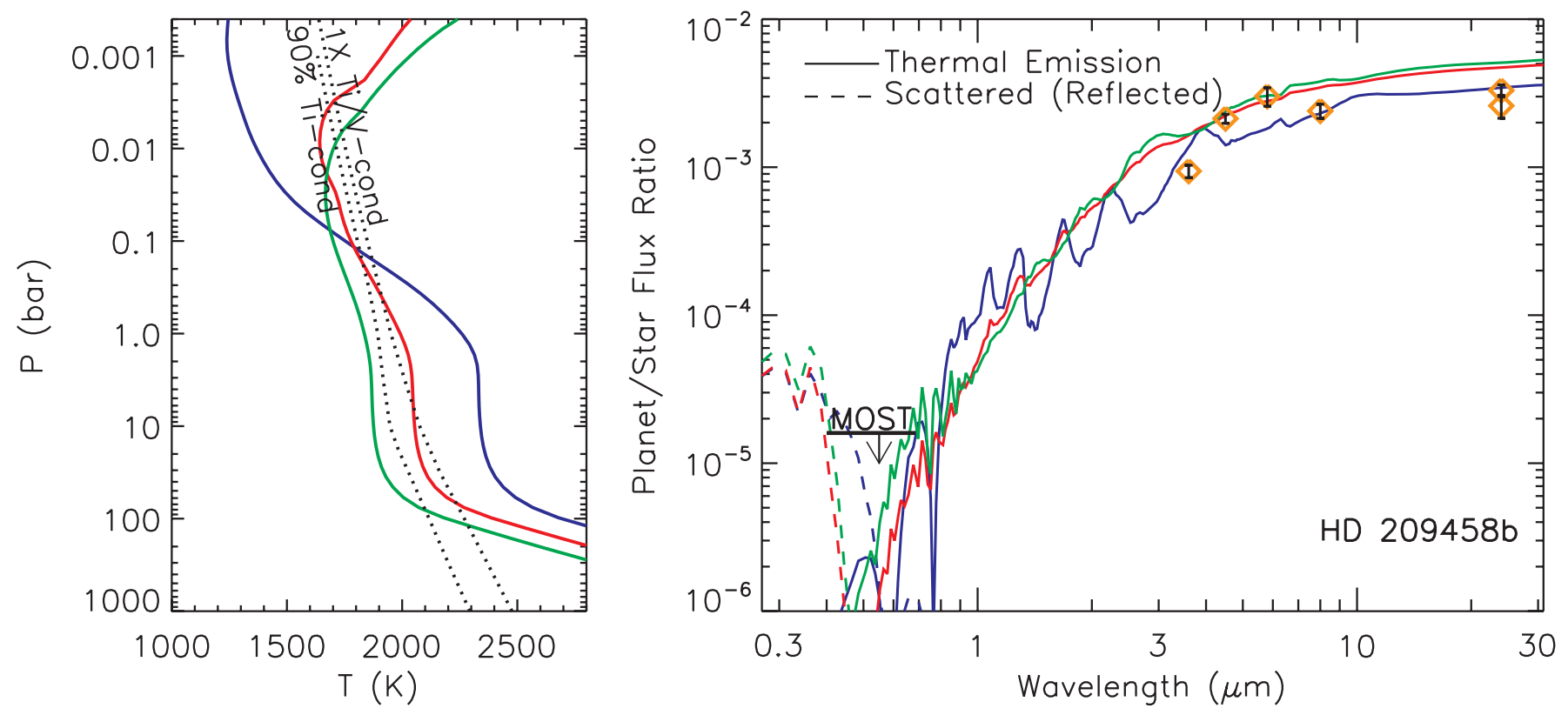

Fig. 12.-Left: HD 209458b atmospheric $P$-T profiles for a model without TiO/VO opacity $($ blue) and two models with TiO/VO opacity ( green and red). The value of $T_{\text {int }}$ is $200 \mathrm{~K}$. Right: Dayside low-resolution model spectra for these profiles, along with observational data. The Knutson et al. (2008) data are shown from 3.6 to $8 \mu \mathrm{m}$. At $24 \mu \mathrm{m}$ the lower point is from Deming et al. (2005) and upper point is from D. Deming et al. (2008, in preparation). Solid lines show thermal emission, while dashed lines show scattered stellar flux. The MOST $1 \sigma$ upper limit from $\sim 0.40-0.68 \mu \mathrm{m}$ from Rowe et al. (2007) is shown as horizontal black line.

upper atmosphere and cooler lower atmosphere for a given planet. The profile that includes $\mathrm{TiO} / \mathrm{VO}$ opacity (red) is cooler than the condensation curve for a solar-abundance mixture of $\mathrm{Ti} / \mathrm{V}$. For this profile, given our equilibrium chemistry table, the abundance of $\mathrm{TiO}$ gas drops even below $10 \%$ of a solar $\mathrm{Ti}$ abundance, but then increases back to solar in the hot upper atmosphere. A proper treatment of the cold trap could leave only a fraction of a solar abundance of $\mathrm{Ti} / \mathrm{V}$ in $\mathrm{TiO} / \mathrm{VO}$ in the upper atmosphere. That being said, a hot stratosphere similar to one that we derive is necessary to explain the planet's spectrum. We think that opacity due to $\mathrm{TiO} / \mathrm{VO}$ is a natural explanation, but more work needs to be done to understand the abundances of these molecules at low pressure in these dynamic atmospheres, such as a nonequilibrium cloud condensation model (see Helling \& Woitke 2006 and $\S 7.1$ ). In green is a similar profile that uses a different method of treating the angular dependence of the incident flux (see Fortney \& Marley 2007). In blue is the day-side profile computed with $\mathrm{TiO} / \mathrm{VO}$ opacity removed.

Figure 12 (right) shows the spectra for these three models. The flux levels at 4.5 and $5.8 \mu \mathrm{m}$ are so large that they can only be caused by a temperature inversion. This is similar to what was found at $8 \mu \mathrm{m}$ for HD 149026 b by Harrington et al. (2007). In the red model the temperature gradient at several millibars is not steep, so the emission features are quite weak. Emission features are more prominent in the green model. Both models with a temperature inversion also fit the $2.2 \mu \mathrm{m}$ constraint from Richardson et al. (2003).

Burrows et al. (2007b) present a nice fit to the Knutson et al. (2008) observations. With their models they explore two additional parameters not found in our models. Most importantly, since they include a sink of energy on the day side, this cools their HD 209458b atmosphere significantly (down to $1000 \mathrm{~K}$ ) at the pressures responsible for emission in the $3.6 \mu \mathrm{m}$ band, leading to much less flux in this band. This enables them to simultaneously fit the 3.6 and $4.5 \mu \mathrm{m}$ band observations. In addition, the opacity source that causes their temperature inversion has an optical depth for absorption that is similar to what they predict for
$\mathrm{TiO} / \mathrm{VO}$, but it is not $\mathrm{TiO} / \mathrm{VO}$ specifically. Similar to our model, Burrows et al. (2007b) have trouble simultaneously fitting the 5.8 and $8.0 \mu \mathrm{m}$ observations. Due to strong opacity caused by water, emission at $24 \mu \mathrm{m}$ emerges from high in the atmosphere (see Fig. 9). In our models, this pressure is within the $2000+\mathrm{K}$ hot stratosphere, whereas Burrows et al. (2007b) finds a cooler upper atmosphere $(\sim 1500 \mathrm{~K})$ above their inversion. In a future work we will explore $P-T$ profiles that extend to lower atmospheric pressure, to better understand how this may effect our derived profiles. The most striking aspect of the Knutson et al. (2008) observations is the relatively cool temperature needed to fit the 3.6 and $8.0 \mu \mathrm{m}$ bands, relative to those at 4.5 and $5.8 \mu \mathrm{m}$. Although our day-side fits show room for improvement, it is exciting that this planet falls into a class with HD 149026b. The details of absorption of incident flux by atmospheres partially depleted in $\mathrm{TiO} / \mathrm{VO}$ due to condensation must be worked out in more detail before it is clear to what degree the $\mathrm{TiO} / \mathrm{VO}$ abundances in the atmosphere of HD 209458b are anomalously high.

HD 209458b is a long-term target for the detection of optical flux from the MOST satellite. Rowe et al. (2007) find a new $1 \sigma$ upper limit to the planet-to-star flux ratio. Figure 12 (right) shows that both models of HD 209458b, with and without a temperature inversion, are consistent with this limit. For the pL class model, this is due to strong optical pressure-broadened lines of $\mathrm{Na}$ and $\mathrm{K}$, as has been expected for some time (Sudarsky et al. 2000; Seager et al. 2000). For the pM class model TiO/VO opacity leads to less scattering of stellar flux, and $\mathrm{TiO} / \mathrm{VO}$ band emission is not bright enough to lead to a detection. It is a bit disappointing that the MOST bandpass $(\sim 400-680 \mathrm{~nm})$ happens to coincide with a minimum in planetary flux. However, the COROT red bandpass and Kepler band extend to redder wavelengths ( $\sim 1000$ and $\sim 850 \mathrm{~nm}$, respectively) such that the detection of planetary optical flux by these satellites for $\mathrm{pM}$ and $\mathrm{pL}$ class planets should be achievable.

The opacity of $\mathrm{TiO} / \mathrm{VO}$ leads to yet another explanation for the weaker than expected sodium absorption feature seen by Charbonneau et al. (2002). Although condensates are still an 


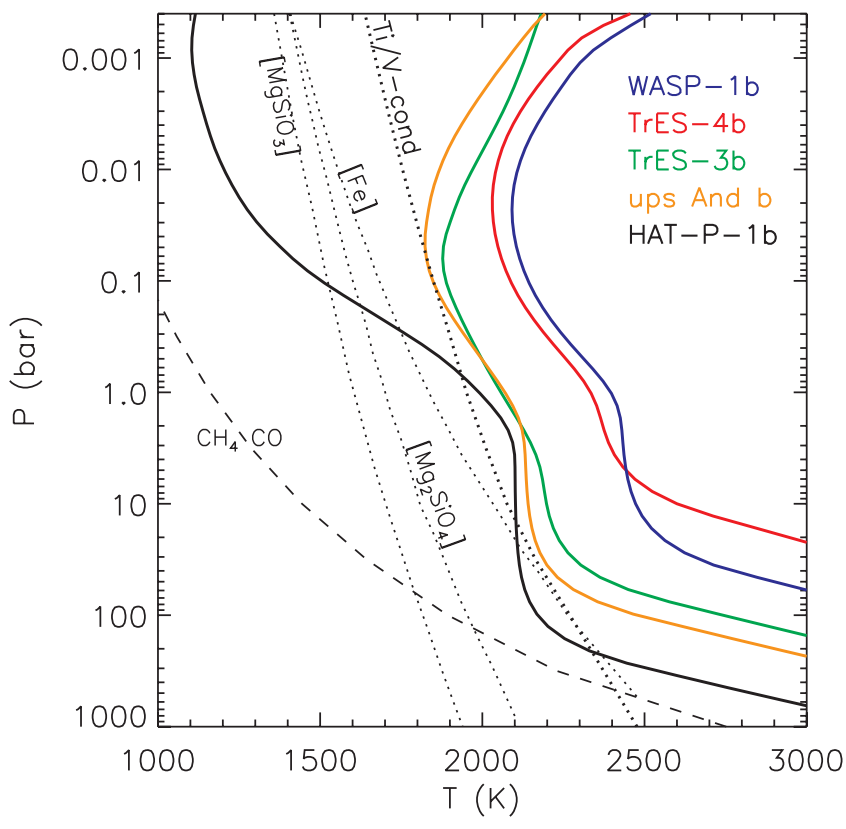

FIG. 13.- Model $P-T$ profiles for five planets. HAT-P-1b is likely pL class, which is the assumption here. The other four planets are pM class.

attractive option (Fortney et al. 2003; Fortney 2005), if TiO/VO are found on the day side of the planet's limb, opacity due to these molecules from $\sim 400$ to $1000 \mathrm{~nm}$ could swamp the expected $\mathrm{Na}$ absorption feature at $589 \mathrm{~nm}$. If the opacity on the limb is everywhere larger in the optical this would lead to a weaker, narrower $\mathrm{Na}$ absorption peak than expected by the first-generation models (Seager \& Sasselov 2000; Brown 2001; Hubbard et al. 2001). This effect can be seen in calculations of Barman et al. (2002), whose description of atmospheric chemistry at that time left addition absorbers of optical flux in the upper atmosphere. It is not clear whether $\mathrm{TiO} / \mathrm{VO}$ would be found in abundance at the planet's limb, but this is an avenue worthy of further exploration.

\subsection{Other Planets}

We can examine the atmospheres of likely $\mathrm{pM}$ class planets from Figure 1. Since the OGLE planets will be difficult to detect in the infrared, we show $P-T$ profiles for WASP-1b (Collier Cameron et al. 2007; Charbonneau et al. 2007), TrES-4b (Mandushev et al. 2007), TrES-3b (O'Donovan et al. 2007), and $v$ And b (Butler et al. 1997; Fuhrmann et al. 1998) in Figure 13. For these profiles the temperature of the adiabatic deep atmosphere at tens to hundred of bars (the top of the interior adiabat) has been chosen to be consistent with the measured planetary radius, assuming a Jupiter/Saturn-like interior abundance of ice/ rock (25 $M_{\oplus}$; see Saumon \& Guillot 2004). The chosen adiabats do not rely on any specific evolution model-for a given planet mass and composition only one particular internal adiabat will match the planet's measured radius (see, e.g., Hubbard et al. 2001 , Fig. 1). For the large-radii transiting planets, deep radiative zones extending down to $\sim 1$ kbar are not consistent with the relatively warm interiors implied by these radii. For $v$ And $b$, which has a mass similar to that of HD 209458b, radius and surface gravity values for HD $209458 \mathrm{~b}$ were used. The temperature of the upper atmosphere of these models are a function of the incident stellar flux, which decreases in magnitude from WASP- $1 \mathrm{~b}$ to $v$ And $\mathrm{b}$. We note that for these hotter $\mathrm{pM}$ class planets, the day-side profiles are everywhere warmer than that required for $\mathrm{Ti} / \mathrm{V}$ condensation, such that opacity due to $\mathrm{TiO} / \mathrm{VO}$
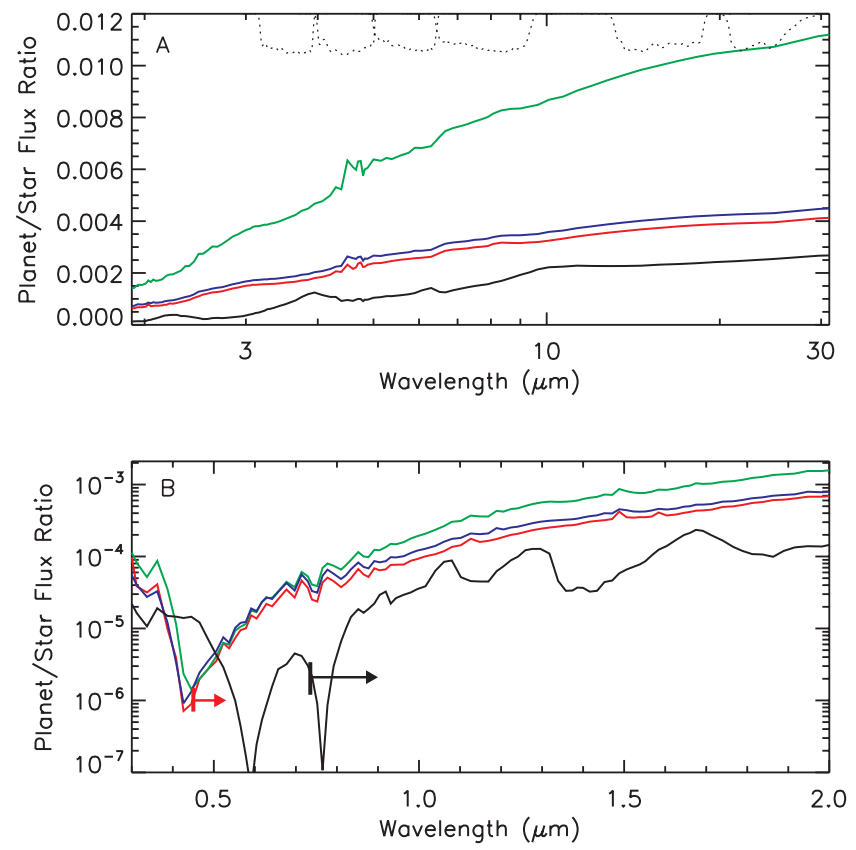

FIG. 14.- - Low-resolution planet-to-star flux ratios for transiting planets shown in Fig. 13. The pM class planets, due to their high incident fluxes are warmer and thus brighter. Flux ratios may approach $10^{-4}$ in the red optical. Weak water emission bands are seen in the mid-infrared for the pM class planets. The bars with arrows indicate the wavelengths at which thermal emitted flux becomes 10 times greater than scattered incident flux, for the HAT-P-1b model (black) and the TrES$3 \mathrm{~b}$ model (red). The $\mathrm{pL}$ class fluxes redward of $\sim 0.7 \mu \mathrm{m}$ are dominated by thermal emission, not reflected light. The pM class fluxes redward of $\sim 0.4 \mu \mathrm{m}$ are dominated by thermal emission.

should not be considered at all anomalous. For comparison we also plot a pL class model for potential transition object HATP-1b (Bakos et al. 2007a; Winn et al. 2007), which transits one member of a main-sequence G star binary system. We highlight it here because the binary nature of the system makes it a good candidate for follow-up observations that rely on relative photometry.

We can examine the computed planet-to-star flux ratios for these models. These are shown in Figure 14, using the same color scheme. Most striking is TrES-3b, whose large planet-tostar radius ratio leads to large flux ratios reaching 0.01 at longer mid-IR wavelengths, as seen in Figure $14 a$. The pM class models have fairly shallow temperature gradients in the hot stratosphere, leading to relatively week emission features in the spectra. A comparison with HAT-P-1b shows that weak water emission features are seen in the pM class planets at wavelengths where HAT-P-1b shows water absorption bands. The feature at $4.5 \mu \mathrm{m}$ is due predominantly to absorption in the stellar atmosphere, along with planetary emission due to $\mathrm{CO}$. The incident stellar spectra are tailored for each planet's parent star and orbital distance, and are taken from Hauschildt et al. (1999).

Figure $14 b$ shows ratios in the optical and near-infrared. As has been pointed out above, for these hot atmospheres thermal emission contributes significantly to the optical flux (Fortney et al. 2005; Seager et al. 2005; López-Morales \& Seager 2007). These pM class planets will be excellent targets for red-optical secondary eclipse observations, as the planet-to-star flux ratio reaches $\sim 10^{-4}$. It is important to note that this optical flux is thermal emission, not scattered incident flux. For the TrES-4b model (red), the vertical bar indicates that by $0.45 \mu \mathrm{m}$, thermal flux is already 10 times greater than reflected light. Thermal flux dominates at all longer optical wavelengths. Even for $\mathrm{pL}$ class 
model for HAT-P-1b, thermal emission is 10 times greater than reflected light at $0.73 \mu \mathrm{m}$. If one wanted to observe true "reflected light" from these atmospheres, the blue spectral regions should be targeted. In a future work we will examine geometric albedos and light curves for these planets to understand current and future data from spacecraft like MOST, Kepler, and COROT. López-Morales \& Seager (2007) have recently investigated prospects for the optical detection of thermal emission from the "very hot Jupiters" in some detail and find the observations promising, even from the ground. They calculate exposure times necessary for detection using blackbody planet models and also those of Hubeny et al. (2003). They highlight OGLE-TR-56b and OGLETR-132b, the hottest planets yet detected, as the most promising candidates for secondary eclipse detections.

\section{PLANETARY CLASSIFICATION}

Previously, Sudarsky et al. $(2000,2003)$ examined the atmospheres and spectra of giant planets as a function of orbital distance and classified these atmospheres in terms of the condensates that may be present. The $\mathrm{pL}$ class that we suggest here overlaps the Sudarsky et al. class IV and V planets, which are both dominated by alkali absorption. Class V planets are distinguished by having silicate and iron clouds in the visible atmosphere. However, it is not yet clear what effect these clouds will have on hot Jupiter spectra and energy balance, because particle sizes, cloud vertical distribution, and even composition $\left(\mathrm{MgSiO}_{3}\right.$ vs. $\mathrm{Mg}_{2} \mathrm{SiO}_{4}$, which have very different optical properties) are not known. Given that the derived $P-T$ profiles and cloud condensation curves for $\mathrm{pL}$ class atmospheres are somewhat parallel (see Fig. 2), there may be a relatively narrow region in the irradiation level (and $T_{\text {eff }}$ ) where clouds are present in the visible atmosphere, rather than down at hundreds of bars. The pM class are planets with atmospheres hotter than those considered by Sudarsky et al.

Although planetary classification based on dominant condensates is certainly attractive, in practice this may by difficult to implement. Clouds are often gray scatters over broad wavelength ranges, so it may be challenging to recognize their presence and definitively identify the composition of a given cloud deck if one is suspected, particularly with data extending over a limited spectral range. In our own solar system the visible clouds of Jupiter and Saturn are surely dominated by ammonia ice, as predicted by equilibrium chemistry condensation, but there is as yet no spectroscopic confirmation of this widely held belief.

A planetary classification scheme based on atomic and molecular absorption features may be more practical to implement and is in keeping with the tradition of basing classification directly on spectral features. This practice would be analogous to the spectral MLT(Y) classification of cool dwarf stars and brown dwarfs. The T spectral type begins when methane is detected in the near-infrared (Kirkpatrick 2005), and it has been suggested that the arrival of ammonia absorption in the near-infrared should delineate the arrival of the Y dwarfs (Kirkpatrick 2005). Young, hot substellar objects and candidate planets directly imaged at wider separations from their parent stars have and certainly will continue to be classified in the standard MLT(Y) scheme (e.g., Chauvin et al. 2005). Thus, extending this classification scheme to all planets, near and far from their parents stars, is a reasonable extension of the status quo. The letters " $M$ " and " $L$ " are used to designate dwarfs at the stellar/substellar boundary according to their spectral features, and for the planetary objects with similar spectroscopic diagnostics as $\mathrm{M}$ and $\mathrm{L}$ dwarfs, we propose to use the spectroscopic classification letters $\mathrm{pM}$ and $\mathrm{pL}$. Classes $\mathrm{pT}$ and $\mathrm{pY}$ would be natural extensions to cooler temperatures.
Planetary classification will surely be complex, as some planets will be dominated by absorbed stellar flux, and others by their own thermal emission, which will yield different $P-T$ profiles at a given $T_{\text {eff }}$. The wavelength ranges at which planets will be most easily observed will also be a function of stellar glare and the sensitivity of future observatories. Planets that are eccentric will straddle two ore more classes, as suggested in Figure 1 for HD 147506b and HD 17156b (see also Sudarsky et al. 2005 for predictions at larger orbital separations). All of these close-in planets will have, at a minimum of complexity, a day/night temperature difference that will make planetary classification difficult. Obviously, mass will be important as well, since with decreasing mass one eventually leaves the gas giant regime. Hansen \& Barman (2007) have pointed out an interesting correlation of the known transiting planets with Safronov number; they appear to fall into two distinct classes. This indicates that classification of planets surely will be complex and multidimensional. Perhaps we will eventually find that the Hansen \& Barman (2007) classes possess different atmospheric abundances due to different accretion histories. Thus, we do not propose a detailed scheme, but rather suggest that the newly available transit data support a method of atmospheric classification based on molecular and atomic absorption, rather than the presence or absence of particular condensates.

\section{DISCUSSION}

\subsection{TiO Chemistry Revisited}

Of course, the boundary between the proposed pM and $\mathrm{pL}$ classes could be somewhat indistinct, as the abundance of $\mathrm{TiO} / \mathrm{VO}$ does not instantaneously drop to zero upon $\mathrm{Ti} / \mathrm{V}$ condensation. For TiO, Figure 2 shows that the mixing ratio falls by a factor of 10 at $\sim 40-180 \mathrm{~K}$ after initial Ti condensation at millibar to bar pressures (A. S. Lee \& K. Lodders 2008, in preparation). The spectra of early L dwarfs show a gradual weakening the $\mathrm{TiO}$ and $\mathrm{VO}$ bands over progressing cooler subclasses (Kirkpatrick et al. 1999; Kirkpatrick 2005); this points to transition planets with depleted but still significant levels of $\mathrm{TiO} / \mathrm{VO}$ whose atmospheric temperatures are $\sim 100-200 \mathrm{~K}$ cooler than the curve of Ti condensation at solar metallicity. HD 209458b is probably near the warm end of this transition region, since HD 189733 b shows no evidence for an inversion. We note that at these same temperatures, $\mathrm{FeH}$ and $\mathrm{CrH}$ are also prominent absorbers at wavelengths blueward of $1.3 \mu \mathrm{m}$ (Kirkpatrick 2005), before being lost to $\mathrm{Fe}$ and $\mathrm{Cr}$ solid. Whether these molecules will eventually help in $\mathrm{pM}$ and $\mathrm{pL}$ subtyping will depend on the quality of data available at these wavelengths.

Since HD 209458b appears to have a temperature inversion even at temperatures where the abundances of $\mathrm{TiO}$ is expected to be waning significantly, for a one-dimensional day-side model, it is natural to inquire whether the abundance of $\mathrm{TiO}$ may differ from the prediction from chemical equilibrium. TiO is typically the major Ti-bearing gas at the $P-T$ conditions where Ti-bearing condensates are expected (Lodders 2002 see). However, $\mathrm{TiO}_{2}$ gas is next in abundance, and other Ti-bearing gases are also present. With decreasing temperature (but before Ti condensation), the TiO gas abundance decreases because abundances of other molecular Ti-bearing gases increase. The abundances of all Ti-bearing gases drop steeply when Ti-bearing condensates form. However, if condensation is kinetically inhibited, the $\mathrm{TiO}$ gas abundances will drop much more gradually with decreasing temperatures, because the $\mathrm{TiO}$ is consumed only by other Ti-bearing gases, not condensates.

The abundance of $\mathrm{TiO}_{2}$ gas is of interest because $\mathrm{TiO}_{2}$ gas is a likely precursor of the $\mathrm{TiO}_{2}$-building blocks in Ti-bearing 
condensates. The net reaction $\mathrm{TiO}+\mathrm{H}_{2} \mathrm{O}=\mathrm{TiO}_{2}+\mathrm{H}_{2}$ is independent of total pressure (there are the same number of molecules on both sides of the reaction). Under the relevant conditions here, the oxidation of the TiO radical is fast. For example, at $1750 \mathrm{~K}$ and $0.01 \mathrm{bar}$, oxidation of $\mathrm{TiO}$ to the equilibrium $\mathrm{TiO}_{2}$ gas abundance takes less than a minute. This means that $\mathrm{TiO}$ abundances are unlikely to be influenced by any vertical mixing processes in planetary or brown dwarf atmospheres, and equilibrium $\mathrm{TiO}$ and $\mathrm{TiO}_{2}$ abundances apply.

The formation of perovskite, $\mathrm{CaTiO}_{3}$, the expected condensate at total pressures $<0.02$ bar in a solar composition gas, requires reaction of $\mathrm{TiO}_{2}$ with $\mathrm{CaO}$. Preliminary calculations show that the oxidation of monatomic $\mathrm{Ca}$ to $\mathrm{CaO}$ gas proceeds on similar timescales as $\mathrm{TiO}$ to $\mathrm{TiO}_{2}$ oxidation. With respect to chemical changes by updraft mixing and quenching it must be emphasized that metal gas phase reactions are very fast, so that equilibrium abundances established at higher temperature regimes will quickly readjust to the appropriate low-temperature equilibria during upwelling. One possibility for HD $209458 \mathrm{~b}$, and planets at similar irradiation levels, is that $\mathrm{TiO}$ is only stable at the (hot) substellar point, but not on the rest of the day side. Another promising avenue for further work may be additional investigation into $\mathrm{V}$ condensation. As discussed in $\S 3.2$, here we assume that VO is depleted with $\mathrm{TiO}$, although strict chemical equilibrium would allow abundant $\mathrm{VO}$ at temperatures $200 \mathrm{~K}$ cooler than needed for initial Ti condensation (Lodders 2002).

At this time it would be unwise to rule out other absorbers, such as nonequilibrium gases or condensates driven by photochemistry, which we do not consider here. As Burrows et al. (2007b) correctly point out, it may be possible for the abundance of a photochemically derived absorber to scale as a function of irradiation level. Liang et al. (2004) have found that methanederived hazes (which are present in the stratospheres of our solar system's giants) would not be stable for a variety of reasons, mainly because methane is not abundant at these high temperatures and low pressures, and haze particulate condensation is prevented. Visscher et al. (2006) also briefly investigated the photochemistry of C- and O-dominated species in HD 209458b. Marley et al. (2007) suggest that photochemistry involving multiple abundant elements, including sulfur, could be important for these planets. Other authors (e.g., Yelle 2004) have investigated the photochemistry of the low-density upper atmosphere of these planets as well. Additional investigations in this area are surely needed.

\subsection{Other Issues}

The models presented here are for day-side average planetary atmospheric structures and spectra. For $\mathrm{pL}$ class planets, redistribution of absorbed energy will likely lead to somewhat cooler atmospheres and lower fluxes than predicted here. The $\mathrm{pM}$ class planetary atmospheres should be very close to a "no redistribution model" that is hottest at the substellar point and becomes cooler toward the planetary limb. A no-redistribution day-side is more luminous than a uniform dayside average (see Barman et al. 2005, who computed planet-wide average, day-side average, and no-redistribution models of HD 209458b and TrES-1). Therefore, our models are guides for understanding, and deviations from the predictions for specific planets are expected. The $\mathrm{pM}$ class planets could potentially be modestly brighter than we have shown.

To our knowledge, no models for the thermal evolution and contraction of $\mathrm{pM}$ class planets have included $\mathrm{TiO} / \mathrm{VO}$ opacity in their atmospheres. Instead, it has been assumed that Ti and $\mathrm{V}$ have condensed into clouds. If it is found that WASP- $1 b, \operatorname{TrES}-4 \mathrm{~b}$, and other $\mathrm{pM}$ class planets have the atmospheric properties that we describe, this will mean that evolution models of these planets will need to be recomputed with new model atmosphere grids. As shown in $\S 4.3$, the atmospheric extension due to the hot stratospheric temperatures and strong $\mathrm{TiO} / \mathrm{VO}$ opacity at optical wavelengths could lead to larger measured radii (see also Burrows et al. 2007b).

\section{CONCLUSIONS: TWO CLASSES OF HOT ATMOSPHERES}

Using one-dimensional radiative-convective equilibrium model atmospheres, we have addressed the class of atmospheres, the pM class, for which $\mathrm{TiO}$ and $\mathrm{VO}$ are extremely strong visible absorbers (Hubeny et al. 2003). This absorbed incident flux drives these planets to have hot $(\sim 2000+K)$ stratospheres. Therefore, these planets will appear very bright in the mid-infrared, with brightness temperatures larger than their equilibrium temperatures. This is the case for HD 149026b (Harrington et al. 2007) and HD 209458b Knutson et al. (2008). In addition, these planets will have large day/night temperature contrasts because radiative time constants at photospheric pressures are much shorter than reasonable advective timescales. The hottest point of the planet should be the substellar point, which absorbs the most flux, leading to perhaps negligible phase shift between the times of maximum measured thermal emission and when the day side is fully visible. This appears to be the situation for planet $v$ And $\mathrm{b}$, observed by Harrington et al. (2006). Given that its irradiation level is intermediate between HD 209458b and HD 149026b, we find that this planet is pM class. Due to the fast radiative times, the day side of these planets may have $P-T$ profiles that do not deviate much from radiative equilibrium models. Although atmospheric dynamics will surely be vigorous, winds will be unable to advect gas before it cools to space. For these planets, high irradiation, the presence of gaseous $\mathrm{TiO}$ and $\mathrm{VO}$, a hot stratosphere, the location of the hottest atmosphere at the substellar point, and a large day/night temperature contrast all go hand in hand.

On the other hand, we have shown that in the pL class, dominated by absorption by $\mathrm{H}_{2} \mathrm{O}, \mathrm{Na}$, and $\mathrm{K}$, photospheric pressures and temperatures prevail such that advective timescales and radiative timescales are similar (see also Seager et al. 2005). Since atmospheric dynamics will be important for the redistribution of energy, the consequences for the structure and thermal emission of these atmospheres will be quite complex. The efficiency of energy redistribution will vary with planetary irradiation level, surface gravity, and rotation rate. Planets of the $\mathrm{pL}$ class will have smaller day/night temperature contrasts and measurable phase shifts in thermal emission light curves that will be wavelength dependent. In addition, these planets may show variability in secondary eclipse depth (e.g., Rauscher et al. 2007a). However, without a better understanding of the dynamics it is difficult to make detailed predictions at this time. Secondary eclipse depths should range somewhere between values expected for a "full redistribution" model and inefficient redistribution. The published secondary eclipse data for $\mathrm{pL}$ class planets TrES-1 and HD $189733 \mathrm{~b}$ are all consistent with this prediction (Fortney et al. 2005; Fortney \& Marley 2007). In addition, the $8 \mu \mathrm{m}$ light curves for $51 \mathrm{Peg} \mathrm{b}$ and HD 209458b (Cowan et al. 2007) and HD 189733b (Knutson et al. 2007) are consistent with this prediction as well. Examination of Figure 1 shows that HD $179949 \mathrm{~b}$ is a pM class planet, and indeed Cowan et al. (2007) found the largest phase variation it their small sample for this planet, but the unknown orbital inclination makes definitive conclusions difficult.

Additional observational results will soon help to test the models presented here. We find that transiting planets WASP-1b, 
TrES- $4 b$, TrES-3b, OGLE-Tr-10b, and TrES- $2 b$ will be in the $\mathrm{pM}$ class, along with nontransiters $v$ And $\mathrm{b}$ and HD 179949. The low-irradiation boundary of this class is not yet clear, and planet HD 209458b shows that temperature inversions persist to irradiation levels where $\mathrm{TiO} / \mathrm{VO}$ are expected to begin being lost to condensation (Burrows et al. 2007b). Perhaps Tio and VO are stable only at the substellar point. At still lower irradiation levels, the limited data for HD 189733b lead us to conclude it is pL class (Fortney \& Marley 2007). Secondary eclipse data for XO-2b, HAT-P-1b, and WASP-2b will be important is determining how temperature inversions (and the $\mathrm{TiO} / \mathrm{VO}$ abundances) wane with irradiation level. Just as in $\mathrm{dM}$ and dL stars, the condensation of Ti and $\mathrm{V}$ is expected to be gradual process, so we fully expect transition objects between the distinct $\mathrm{pM}$ and $\mathrm{pL}$ class members.

We will soon have additional information that will help shed light on the atmosphere of HD 209458b. The $8 \mu \mathrm{m}$ light curve for HD 209458b obtained by Cowan et al. (2007) shows little phase variation. However, if our theory connecting $\mathrm{TiO} / \mathrm{VO}$ opacity and temperature inversions to large day/night contrasts is correct, we expect to see a large variation. Soon $\mathrm{H}$. Knutson and collaborators will obtain half-orbit light curves for HD $209458 \mathrm{~b}$ at 8 and $24 \mu \mathrm{m}$. The quality should be comparable to that obtained by Knutson et al. (2007) for HD 189733b and will put our theory to the test. HD 147506, with an eccentricity of 0.517 (Bakos et al. 2007b), and HD 17156, with an eccentricity of 0.67 (Fischer et al. 2007; Barbieri et al. 2007), will be extremely interesting cases as the flux they receive varies by factors of 9 and 26, respectively, between periapse and apoapse. They should each spend part of their orbits as pL class and part as pM class. This makes predictions difficult, but large day/night temperatures differences at apoapse are likely.

There has recently been considerable discussion on the relative merits of multidimensional dynamical models and onedimensional radiative-convective model atmospheres for these highly irradiated planets. Both kinds of studies provide interesting predictions. While it could be claimed that one-dimensional radiative-convective models are unrealistic because they "lack dynamics," they do include very detailed chemistry, vast opacity databases, and advanced nongray radiative transfer, which all dynamics models for these planets lack. Given the large diversity in predictions among the various dynamical models, which use a host of simplifications, the next step will be combining dynamics and radiative transfer, an idea which has been mentioned or advanced by a number of authors (Seager et al. 2005; Barman et al. 2005; Fortney et al. 2006a; Burrows et al. 2006; Dobbs-Dixon \& Lin 2008). Eventually we will be able to give up one-dimensional $f$-type parameters to treat the incident flux in a more realistic fashion for these exotic atmospheres. We recently started working toward this goal in Fortney et al. (2006a; see also Dobbs-Dixon $\&$ Lin 2008); work continues, and we believe it has a promising future. We think that the predictions we have made here with a one-dimensional model will provide a framework for understanding the observations to come. Additional observations for both $\mathrm{pL}$ and $\mathrm{pM}$ class planets, along with additional theoretical and modeling efforts, should further clarify our understanding.

We thank Adam Showman for valuable discussions and for help with the calculation of radiative time constants. We thank Didier Saumon for providing the data files for Figure 3. Jason Rowe kindly provided us with the MOST geometric albedo upper limit for HD 209458b in advance of publication. We also thank the referee and Jason Barnes for comments that improved the draft. J. J. F. acknowledges the support of a Spitzer Fellowship from NASA. Work by K. L. is supported by NSF grant AST 0406963 and NASA grant NNG06GC26G. Work by M. S. M. is supported by the NASA Planetary Atmospheres Program.
Ackerman, A. S., \& Marley, M. S. 2001, ApJ, 556, 872

Allard, F., Hauschildt, P. H., Alexander, D. R., Tamanai, A., \& Schweitzer, A. 2001, ApJ, 556, 357

Appleby, J. F., \& Hogan, J. S. 1984, Icarus, 59, 336

Bakos, G. Á., et al. 2007a, ApJ, 656, 552 2007b, ApJ, 670, 826

Baraffe, I., Chabrier, G., Barman, T. S., Allard, F., \& Hauschildt, P. H. 2003 , A\&A, 402, 701

Barbieri, M., et al. 2007, A\&A, 476, L13

Barman, T. 2007, ApJ, 661, L191

Barman, T. S., Hauschildt, P. H., \& Allard, F. 2005, ApJ, 632, 1132

Barman, T. S., Hauschildt, P. H., Schweitzer, A., Stancil, P. C., Baron, E., \& Allard, F. 2002, ApJ, 569, L51

Bouchy, F., Pont, F., Santos, N. C., Melo, C., Mayor, M., Queloz, D., \& Udry, S. 2004, A\&A, 421, L13

Bouchy, F., et al. 2005, A\&A, 444, L15

Brown, T. M. 2001, ApJ, 553, 1006

Burrows, A., Hubeny, I., Budaj, J., \& Hubbard, W. B. 2007a, ApJ, 661, 502

Burrows, A., Hubeny, I., Budaj, J., Knutson, H. A., \& Charbonneau, D. 2007b, ApJ, 668, L171

Burrows, A., Hubeny, I., Hubbard, W. B., Sudarsky, D., \& Fortney, J. J. 2004, ApJ, 610, L53

Burrows, A., Marley, M. S., \& Sharp, C. M. 2000, ApJ, 531, 438

Burrows, A., \& Sharp, C. M. 1999, ApJ, 512, 843

Burrows, A., Sudarsky, D., \& Hubbard, W. B. 2003, ApJ, 594, 545

Burrows, A., Sudarsky, D., \& Hubeny, I. 2006, ApJ, 650, 1140

Burrows, A., et al. 1997, ApJ, 491, 856

Butler, R. P., Marcy, G. W., Williams, E., Hauser, H., \& Shirts, P. 1997, ApJ, 474, L115

Chabrier, G., \& Baraffe, I. 2007, ApJ, 661, L81

Chamberlain, J. W., \& Hunten, D. M. 1987, Theory of Planetary Atmospheres: An Introduction to Their Physics and Chemistry (2nd. ed.; Orlando: Academic Press)
REFERENCES

Charbonneau, D., Brown, T. M., Noyes, R. W., \& Gilliland, R. L. 2002, ApJ, 568,377

Charbonneau, D., Winn, J. N., Everett, M. E., Latham, D. W., Holman, M. J., Esquerdo, G. A., \& O’Donovan, F. T. 2007, ApJ, 658, 1322

Charbonneau, D., et al. 2005, ApJ, 626, 523

Chauvin, G., Lagrange, A.-M., Dumas, C., Zuckerman, B., Mouillet, D., Song, I.,

Beuzit, J.-L., \& Lowrance, P. 2005, A\&A, 438, L25

Collier Cameron, A., et al. 2007, MNRAS, 375, 951

Cooper, C. S., \& Showman, A. P. 2005, ApJ, 629, L45

2006, ApJ, 649, 1048

Cowan, N. B., Agol, E., \& Charbonneau, D. 2007, MNRAS, 379, 641

Cushing, M. C., et al. 2008, ApJ, 678, 1372

Deming, D., Harrington, J., Laughlin, G., Seager, S., Navarro, S. B., Bowman, W. C., \& Horning, K. 2007, ApJ, 667, L199

Deming, D., Harrington, J., Seager, S., \& Richardson, L. J. 2006, ApJ, 644, 560

Deming, D., Seager, S., Richardson, L. J., \& Harrington, J. 2005, Nature, 434, 740

Demory, B.-O., et al. 2007, A\&A, 475, 1125

Dobbs-Dixon, I., \& Lin, D. N. C. 2008, ApJ, 673, 513

Fischer, D. A., et al. 2007, ApJ, 669, 1336

Fortney, J. J. 2005, MNRAS, 364, 649

Fortney, J. J., Cooper, C. S., Showman, A. P., Marley, M. S., \& Freedman, R. S. 2006a, ApJ, 652, 746

Fortney, J. J., \& Marley, M. S. 2007, ApJ, 666, L45

Fortney, J. J., Marley, M. S., Lodders, K., Saumon, D., \& Freedman, R. 2005, ApJ, 627, L69

Fortney, J. J., Saumon, D., Marley, M. S., Lodders, K., \& Freedman, R. S. 2006b, ApJ, 642, 495

Fortney, J. J., Sudarsky, D., Hubeny, I., Cooper, C. S., Hubbard, W. B., Burrows, A., \& Lunine, J. I. 2003, ApJ, 589, 615

Freedman, R. S., Marley, M. S., \& Lodders, K. 2008, ApJS, 174, 504

Fuhrmann, K., Pfeiffer, M. J., \& Bernkopf, J. 1998, A\&A, 336, 942

Goody, R. M., \& Yung, Y. L. 1989, Atmospheric Radiation: Theoretical Basis (2nd ed.; New York: Oxford Univ. Press) 
Grillmair, C. J., Charbonneau, D., Burrows, A., Armus, L., Stauffer, J., Meadows, V., Van Cleve, J., \& Levine, D. 2007, ApJ, 658, L115

Hansen, B. M. S., \& Barman, T. 2007, ApJ, 671, 861

Harrington, J., Hansen, B. M., Luszcz, S. H., Seager, S., Deming, D., Menou, K., Cho, J. Y.-K., \& Richardson, L. J. 2006, Science, 314, 623

Harrington, J., Luszcz, S., Seager, S., Deming, D., \& Richardson, L. J. 2007, Nature, 447, 691

Hauschildt, P. H., Allard, F., Ferguson, J., Baron, E., \& Alexander, D. R. 1999, ApJ, 525, 871

Helling, C., \& Woitke, P. 2006, A\&A, 455, 325

Hubbard, W. B., Fortney, J. J., Lunine, J. I., Burrows, A., Sudarsky, D., \& Pinto, P. 2001, ApJ, 560, 413

Hubeny, I., Burrows, A., \& Sudarsky, D. 2003, ApJ, 594, 1011

Iro, N., Bezard, B., \& Guillot, T. 2005, A\&A, 436, 719

Kirkpatrick, J. D. 2005, ARA\&A, 43, 195

Kirkpatrick, J. D., et al. 1999, ApJ, 519, 802

Knutson, H. A., Charbonneau, D., Allen, L. E., Burrows, A., \& Megeath, S. T. 2008, ApJ, 673, 526

Knutson, H. A., et al. 2007, Nature, 447, 183

Konacki, M., Torres, G., Jha, S., \& Sasselov, D. D. 2003, Nature, 421, 507

Kornacki, A. S., \& Fegley, B. J. 1986, Earth Planet. Sci. Lett., 79, 217

Langton, J., \& Laughlin, G. 2007, ApJ, 657, L113

Liang, M., Seager, S., Parkinson, C. D., Lee, A. Y.-T., \& Yung, Y. L. 2004, ApJ, 605, L61

Lodders, K. 1999, ApJ, 519, 793

. 2002, ApJ, 577, 974

$$
\text { 2003, ApJ, 591, } 1220
$$

Lodders, K., \& Fegley, B. 2002, Icarus, 155, 393

2006, Astrophysics Update 2 (Berlin: Springer, 2006)

López-Morales, M., \& Seager, S. 2007, ApJ, 667, L191

Mandushev, G., et al. 2007, ApJ, 667, L195

Marley, M. S., Fortney, J., Seager, S., \& Barman, T. 2007, in Protostars and Planets V, ed. B. Reipurth, D. Jewitt, \& K. Keil (Tucson: Univ. Arizona Press), 733

Marley, M. S., \& McKay, C. P. 1999, Icarus, 138, 268

Marley, M. S., Saumon, D., Guillot, T., Freedman, R. S., Hubbard, W. B., Burrows, A., \& Lunine, J. I. 1996, Science, 272, 1919

Marley, M. S., Seager, S., Saumon, D., Lodders, K., Ackerman, A. S., Freedman, R. S., \& Fan, X. 2002, ApJ, 568, 335
McKay, C. P., Pollack, J. B., \& Courtin, R. 1989, Icarus, 80, 23

O'Donovan, F. T., et al. 2007, ApJ, 663, L37

Rauscher, E., Menou, K., Cho, J. Y.-K., Seager, S., \& Hansen, B. M. S. 2007a, ApJ, 662, L115

Rauscher, E., Menou, K., Seager, S., Deming, D., Cho, J. Y.-K., \& Hansen, B. M. S. 2007b, ApJ, 664, 1199

Richardson, L. J., Deming, D., Horning, K., Seager, S., \& Harrington, J. 2007, Nature, 445, 892

Richardson, L. J., Deming, D., \& Seager, S. 2003, ApJ, 597, 581

Rowe, J. F., et al. 2007, ApJ, submitted (arXiv:0711.4111)

Salby, M. L. 1996, Fundamentals of Atmospheric Physics (San Diego: Academic Press)

Saumon, D., \& Guillot, T. 2004, ApJ, 609, 1170

Saumon, D., Marley, M. S., Cushing, M. C., Leggett, S. K., Roellig, T. L., Lodders, K., \& Freedman, R. S. 2006, ApJ, 647, 552

Saumon, D., et al. 2007, ApJ, 656, 1136

Seager, S., Richardson, L. J., Hansen, B. M. S., Menou, K., Cho, J. Y.-K., \& Deming, D. 2005, ApJ, 632, 1122

Seager, S., \& Sasselov, D. D. 2000, ApJ, 537, 916

Seager, S., Whitney, B. A., \& Sasselov, D. D. 2000, ApJ, 540, 504

Sharp, C. M., \& Burrows, A. 2007, ApJS, 168, 140

Showman, A. P., \& Guillot, T. 2002, A\&A, 385, 166

Stevenson, D. J. 1985, Icarus, 62, 4

Sudarsky, D., Burrows, A., \& Hubeny, I. 2003, ApJ, 588, 1121

Sudarsky, D., Burrows, A., Hubeny, I., \& Li, A. 2005, ApJ, 627, 520

Sudarsky, D., Burrows, A., \& Pinto, P. 2000, ApJ, 538, 885

Swain, M. R., Bouwman, J., Akeson, R., Lawler, S., \& Beichman, C. 2008, ApJ, 674, 482

Tinetti, G., et al. 2007, Nature, 448, 169

Toon, O. B., McKay, C. P., Ackerman, T. P., \& Santhanam, K. 1989, J. Geophys. Res., 94, 16287

Visscher, C., Lodders, K., \& Fegley, B. J. 2006, ApJ, 648, 1181

Williams, P. K. G., Charbonneau, D., Cooper, C. S., Showman, A. P., \& Fortney, J. J. 2006, ApJ, 649, 1020

Winn, J. N., et al. 2007, AJ, 134, 1707

Yelle, R. V. 2004, Icarus, 170, 167 